\title{
Beobachtungen über den Nahrungserwerb bei der Naticide Lunatia nitida Donovan (Gastropoda Prosobranchia)
}

\author{
Von Erich Ziegelmeier \\ Biologische Anstalt Helgoland, List auf Sylt, in der Bundesforschungsanstalt für Fischerei \\ (Mit 15 Abbildungen und 1 Tabelle) \\ Inhaltsübersicht \\ A. Einleitung S. 1 - B. Material und Methode S. 4 - C. Das Verhalten von Lunatia \\ nitida im Aquarium und Beobachtungen über die Nahrungssuche S. $5-$ D. Beobachtungen \\ an den von Lunatia im Aquarium gebohrten Löchern S. 8 - I. Lage der Bohrlöcher auf \\ den Muschelschalen S. 9 - II. Die Form und Beschaffenheit der Bohrlöcher S. 11 - 1. Fertige \\ Bohrungen S. 11 - 2. Unfertige Bohrungen S. 13 - E. Direkte Beobachtung des Bohr- \\ vorganges S. 14 - I. Die Herstellung der Bohrlöher S. 16 - II. Dauer des Bohraktes \\ S. 18 - III. Der Freßakt S. 19 - F. Bau und Funktion des Lunatia-Rüssels S. 20 - \\ I. Der Bau S. 20 - II. Zusammenwirken der Pharynxteile S. 26 - 1. Beim Bohren S. 26 - \\ 2. Beim Freßakt S. 27 - III. Die "Bohrdrüse“ S. 28 - G. Zusammenfassung S. 31 - \\ H. Literatur S. 32.
}

\section{A. Einleitung}

Eine besondere Art des Nahrungserwerbs finden wit bei den marinen carnivoren Gastropoden, die ihre beschalten Beutetiere anbohren und den Weichkörper durch das Bohrloch ausfressen. Zu den Bohrern unter den Raubschnecken gehören die Naticidae und Muricidae. Die Vertreter beider Familien sind befähigt, mit ihrem Rüssel in die Kalkschalen von Muscheln und Schnecken kleine, mehr oder weniger kreisrunde Löcher zu bohren, die bei den Muricidae ein fast zylindrisches, steilwandigeres Aussehen haben und bei den Naticidae konisch beschaffen sind, wobei die Wandungen eine Kehlung besitzen. Während die Muricidae Tiere der Epifauna anfallen, gelegentlich aber auch Aas annehmen, sind die im Boden lebenden echten "Jäger" unter den Raubschnecken, die Naticidae, über deren Bohrweise in der vorliegenden Darstellung berichtet werden soll, auf lebende Beute angewiesen.

Obgleich diese Art des Nahrungserwerbs seit langem bekannt ist, und die angebohrten Molluskenschalen in Bodengreifer- und Dredschmaterial, auch angespült am Strand, mitunter häufig zu finden sind, konnte bisher nicht geklärt werden, wie die Schnecken die Bohrlücher herstellen, da eine direkte Beobachtung des Bohrvorganges noch nicht geglüdkt war. Bei Natica ist dies besonders schwierig, da sie ihre Opfer stets im Boden anbohrt und ausfriBt. Bis auf einige experimentelle Arbeiten, von denen die Untersuchungen von Schiemenz (1891), Hrrsch (1915), P.-H. Fischer (1922), Boettger (1930) und Ankel (1937) die wichtigsten sind, stützen sich eine Reihe anderer Autoren vor allem auf die Beobadhtungen an durchbohrten Schalen, wobei aus der Lage der Bohrungen, der Form und Struktur der Wandungen der fertigen und unvollständigen Bohrlöcher, auf die Bohrtätigkeit geschlossen wurde.

So sollen nach den zahlreichen Angaben in der Literatur, von der nur die wesentliche genannt ist, die Bohrlöcher zustande kommen:

1. Durch Atzung - Schremenz, Hirsch, Boetrger, Livan (1937), Ankel (1936a, 1937, $1938 \mathrm{a}, 1938 \mathrm{~b})$. 
2. Mechanisch mit Hilfe der Radula - P. H. Fischer, Pelseneer (1925), Loppens (1927), JENSEN (1951).

3. Durch eine Kombination, bei der die Tätigkeit der Radula unterstützt wird durch die Abscheidung eines kalklösenden Sekrets - angedeutet von Simroth (1896-1907) und P.-H. FisCHER.

Schiemenz sah in der "Bohrdrüse", einer bei den Naticiden hinter der Mundöffnung auf der Unterseite des Rüssels liegenden tellerförmigen Scheibe, ein Organ, das bei ausgestrecktem Rüssel auf einer Muschel oder Schnecke liegend, durch Absonderung einer Säure die Schalen der Beutetiere durchätzt. ANKEL (1937) erzielte mit frisch herauspräparierten Bohrdrüsen von Lunatia nitida (DoNovan) auf der Innenseite von Trivita-Sdalen Atzwirkungen, die er einer abgeschiedenen Säure zuschrieb. Uber die chemische Natur des Bohrdrüsensekrets beridhtet später ANkri (1938b), daß eine Analyse des vermutlich wirksamen Stoffes in der Bohrdrüse von Natica millepunctata auf Schwefelsäure, Phosphorsäure, Salpetersäure und Ameisensäure, „wie überhaupt jede Prüfung auf das Vorkommen einer freien Säure" negativ ausfiel. Ankes erwähnt eine gesprächsweise Mitteilung von G. C. HiRsch, der vermutete, daß "bei der Lösung des Kalkes durch Natica ein spezifisches Ferment, eine Calcase, eine entscheidende Rolle" spielen könnte. Als Beweise für die Deutung der Bohrtätigkeit mit chemischen Mitteln werden von verschiedenen Autoren meist Atzwirkungen an den Wandungen der Bohrlöcher und die Ubereinstimmung der Größen von Bohrdrüse und Bohrloch angeführt.

Die Gegner der chemischen Erklärung für den Bohrakt begründen ihre Ansicht, daß nur die Radula beim Bohrvorgang tätig ist,

1. mit Beobachtungen von Strukturveränderungen an den Lochrändern, die auf Raspelspuren der Radulazähne hindeuten und

2. mit dem Argument, daß die von der Bohrdrüse produzierte Menge an kalklösenden Mitteln zu gering und in ihrer Wirkung zu schwach sei, um in der verhältnismäßig kurzen Zeit eine Molluskenschale durchzuätzen.

JENSEN kommt auf Grund von Beobachtungen an Bohrlöchern in Rocheneikapseln von Raja fyllae und Raja radiata aus der Davis-Straße (Tiefe $460 \mathrm{~m}$ ) zu dem Schluß, daß diese Bohrungen

1. wegen ihrer für die Naticidae typischen Form,

2. wegen der chemischen Zusammensetzung der hornigen, widerstandsfähigen Kapselwände und

3. wegen der Kratzspuren auf dea Bohrlochwandungen

von den in diesem Gebiet vorkommenden carnivoren Gastropodenspezies Natica affinis (LüTk.) und Natica pallida (Don.) nicht mit chemischen Mitteln, sondern mechanisch mit der Radula, möglicherweise mit den Kiefern hergestellt worden sind.

Die Frage, ob die Bohrdrüse oder die Radula die Werkzeuge sind, mit denen Natica bohrt, kann aber erst beantwortet werden, wenn es gelingt, die Schnecken während des Bohraktes zu beobachten. Für die kleine Naticide, Lunatia nitida (Donovan), mit der auch ANKEL (1937) seine Atzversuche durchführte, konnte durch Aquariumsversuche nachgew:esen werden, daß sie ihre Beute mit der Radula anbohrt und den Weichkörper durch das Bohrloch ausfrißt. Es ist anzunehmen, daß dies auch für die anderen Spezies der Naticidae zutrifft.

Anlaf zu den Versuchen gaben Beobachtungen während der quantitativen Bodengreiferuntersuchungen ab Herbst 1949 in der Deutschen Bucht: Nach der Zunahme von Muscheln, die im Herbst 1950 und Frühjahr 1951 ein Maximum zeigten, stiegen die Bestandsziffern der im Untersuchungsgebiet am häufigsten vorkommenden $\mathcal{N}$ atica-Art, Lunatia nitida.

Ab Herbst 1951 waren die Schnecken auf fast allen Stationen, unabhängig von der Bodenbeschaffenheit, vom sandigen Schlick bis zum reinen Sand, im Bodengreifer ( $1 / 10 \mathrm{qm}$ ) zu finden (Abb. 1). Besonders auffällig zeigte sich die Abhängigkeit in einer Massensiedlung von Aloidis gibba (OLIvI) mit dem Kerngebiet etwa $7 \mathrm{Sm}$ nordwestlich von Helgoland (folg. Tab.).

Die höhere Abundanz von Lunatia nitida ließ sich vor allem an den zahlreicher angebohrten Schalen im autochthonen Schill der betreffenden Proben erkennen. Diese Feststellung gab schließlich den unmittelbaren Anlaß zur Untersuchung des Bohrproblems bei den Naticiden. Es schien nämlich, als ob 


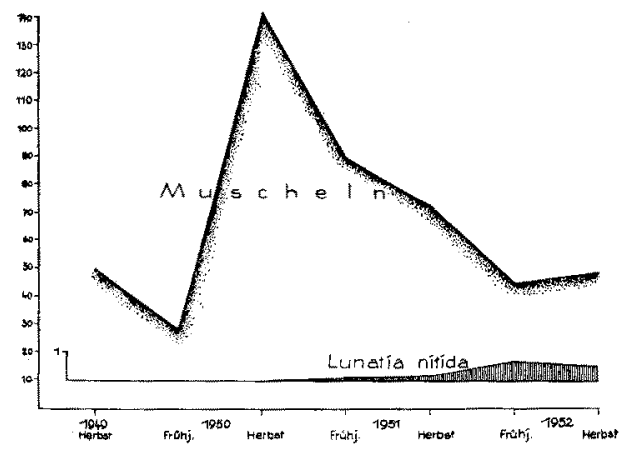

Abb. 1. Graphische Darstellung der Bestandszahlen von Muscheln und Lunatia nitida nach quantitativen Bodengreiferuntersuchungen in der Deutschen Bucht für die Zeit von Herbst 1949 bis Herbst 1952. Die Ordinate gibt die Anzahl auf 1/10 $\mathrm{qm}$ im Durchschnitt aus sämtlichen Stationen und Bodengreiferfängen für die einzelnen Terminfahrten an.

\begin{tabular}{lcccccccc} 
& \multicolumn{1}{c}{1949} & \multicolumn{2}{c}{1950} & \multicolumn{2}{c}{1951} & \multicolumn{2}{c}{1952} \\
& Herbst & Frühj. & Herbst & Frühj. & Herbst & Frühj. Herbst \\
\hline $\begin{array}{l}\text { Aloidis gibba Anzahl auf } \\
1 / 10 \text { qm (Durchschnitt aus } \\
2 \text { Bodengreiferproben) }\end{array}$ & 17 & 21 & 273 & 238 & 123 & 71 & 26 \\
\hline $\begin{array}{l}\text { Lunatia nitida Anzahl auf } \\
\begin{array}{l}1 / 10 \text { qm (Durchschnitt aus } \\
2 \text { Bodengreiferproben) }\end{array}\end{array}$ & - & Keine & - & 1 & 3 & 5 & 2 \\
\hline
\end{tabular}

dünnschalige Muscheln weniger angebohrt werden als Tiere mit dicken Schalen. So konnten oft massenhaft Klappen der dickschaligen Aloidis gibba sowie Spisula subtruncata und $S p$. solida mit oft mehreren vollständigen und unfertigen Bohrungen (bei Aloidis zuweilen bis zu 5 auf einer Klappe) aus dem Siebrest herausgesucht werden, während von den dünnschaligen Abra- und Angulusarten nur gelegentlich Schalen zu finden waren, die einwandfrei erkennbare Bohrlöcher besaßen. Diese Beobachtung ließ die Vermutung aufkommen, daß Lunatia bestimmte Muschelarten als Nahrung bevorzugt und daß vielleicht ein Zusammenhang zwischen der Schalendicke und der Bohrweise besteht. Wie aber die zu dem Zweck angesetten Aquariumsversuche zeigten, trifft dies nicht zu. Sämtliche Muschelarten, die als Nahrungstiere bei den Untersuchungen verwandt wurden, sind angebohrt und ausgef ressen worden. Weniger angebohrt, z. T. verschmäht wurden lediglich die Muscheln mit einer skulptierten Schalenoberfläche, wie Venus gallina und Cardium edule, worüber in der vorliegenden Darstellung noch zu berichten sein wird. Der Grund, weshalb im Siebrest weniger dünne Klappen mit Bohrlöchern zu finden sind, ist wohl darin zu suchen, daß viele der angebohrten dünnen Schalen, wie z. B. der im Sandboden lebenden Angulus fabula, die an dem oben erwähnten Muschel-Maximum im Herbst 1950 und Frühjahr 1951 wesentlichen Anteil hat, infolge der ständigen Sedimentbewegung häufig an den Bohrstellen beschädigt werden oder zerbrechen, so daß die Bohrungen als solche nicht mehr zu erkennen sind.

Für die Beurteilung der Bodentierbestände und die Kenntnis der Faktoren, welche Bestandsschwankungen verursachen, ist im Hinblick auf das Vorkommen von Lunatia, neben der Frage der Bevorzugung bestimmter Muschelarten als Nährtiere, weiterhin wissenswert, in welchem Maße der als Schollen- 
nahrung wichtige Muschelbestand durch die Raubschnecken dezimiert wird. Um hierüber etwas aussagen zu können, ist es notwendig, die für den Bohr und Freßakt benötigte Zeit zu kennen, die aber wiederum erst durch die bereits erwähnte direkte Beobachtung der Nabelschnecken beim Bohren und Fressen ermittelt werden kann. So führten im Rahmen der quantitativen Bodengreiferuntersuchungen die wegen der beiden angeführten ökologischen Fragestellungen im Sept. 1952 begonnenen Aquariumsversuche schließlich dazu, das Verhalten von Lunatia nitida beim Nahrungserwerb, vom Annehmen der Muschel bis zum vollkommen aufgezehrten Weichkörper unmittelbar zu beobachten und zur. Kenntnis des Baues und der Funktion des beim Bohr- und Freßakt ausschließlich beteiligten Rüssels dieser Naticidenspezies beizutragen.

\section{B. Material und Methode}

Die Schnecken sind lebend hauptsächlich aus den Bodengreiferproben während der Terminfahrten mit F. K. "Uthörn" in der Deutschen Bucht herausgesucht worden. Gelegentlich waren einige Tiere im Beifang eines Hols mit dem Helgoländer Trawl zu finden. An Deck hielten sich die Schnecken in Gläsern, in die eine etwa $1-2 \mathrm{~cm}$ dicke Sedimentschicht geschüttet wurde, bei häufigerem Wasserwechsel längere Zeit sehr gut; in die Gläser mit eingesetgte lebende Muscheln bohrte Lunatia troty der Bewegungen des Schiffes und der ständigen Vibration durch die Maschine bereits während der Fahrt an.

Im Laboratorium wurden die Tiere in $30 \mathrm{~cm}$ hohen Aquarien (Grundfläche $20 \times 20 \mathrm{~cm})$ bei stetiger Durchlüftung gehältert. Der Wasserwechsel in den Aquarien erfolgte im Abstand von etwa 3 Monaten. Zur Eingewöhnung der Tiere wurde in die Becken zuerst eine etwa $5-7 \mathrm{~cm}$ hohe Sedimentschicht von den Fundorten gebracht und später gegen eine $2-3 \mathrm{~cm}$ dicke Schicht gut ausgewaschenen, reinen, feinen Sandes ausgetauscht, was sich für die bessere Durchlüftung des Bodens als sehr vorteilhaft erwies. So gehälterte Tiere hielten sich bis zu 7 Monaten und konnten jederzeit zu den Versuchen und Beobachtungen benutgt werden. Etwa 3 Wochen (nach dem Einsetgen) blieben die Schnecken ohne Nahrung. Als Futtermuscheln wurden während der Versuche folgende Arten verwandt: Nucula nitida, $\mathcal{N}$. nucleus, Montacuta ferruginosa, Cardium edule, Venus gallina, Macoma baltica, Angulus tenuis, A. fabula, Abra alba, Spisula subtruncata, Sp. solida, Aloidis gibba, Mya arenaria juv.

Die Temperatur hatte bei der Hälterung nur geringen Einfluß auf die Schnecken. Gegen Temperaturschwankungen ist Lunatia nitida unempfindlich, Wassertemperaturunterschiede innerhalb eines Tages in den Becken, die im geheizten Zimmer standen, von $13^{\circ} \mathrm{C}$ konnten von den Tieren ohne Schädigung ertragen werden. Die tiefste Wassertemperatur während der Untersuchungszeit betrug $+1{ }^{\circ} \mathrm{C}$ (1. Hälfte Februar 1953), die höchste $23^{\circ} \mathrm{C}$. Nur bei Werten über $20^{\circ} \mathrm{C}$ zeigten sich die Schnecken träge, und stark hungernde Tiere nahmen keine Muscheln an, die sonst sofort überwältigt wurden.

Für die Beobachtung unter dem Binokular und für die photographischen Aufnahmen ${ }^{1}$ ) sind die Schnecken mit ihren Beutetieren in kleine Küvetten

1) Sämtliche Photos mit Ausnahme von Abb. $6 \mathrm{~A}$ und den Mikroaufnahmen, sind mit dem Leitz-Elmar $1: 3,5, f=5 \mathrm{~cm}$ hergestellt worden. Das Objektiv wurde verwandt: 1 . in der Leica-Kamera in Verbindung mit dem Hilfsgerät „BEHOO“ für Nahaufnahmen und 2. mit einer Reisekamera $13 \times 18$. Als Lichtquelle wurden benutzt: Monla-Lampe, Synchroblitzer und Bogenlampe. (Sämtl. Leitz, Wetzlar.) 
oder kleine Flachbecken gesetyt worden. Im einzelnen wird bei der Besprechung der Versuche auf die dabei angewandten Methoden noch näher eingegangen. Allgemein ist zur Methodik noch zu bemerken, daß, nach den anfänglichen Versuchen zur Frage einer evtl. Bevorzugung bestimmter Muschelarten als Nahrungstiere, zur Ermöglichung der unmittelbaren Beobachtung die Schnecken 1. durch die langsame Eingewöhnung in die besonderen Lebensbedingungen, 2. durch Einschieben von bestimmten Hungerzeiten, 3. Beobachtung über Menge und Größe der als Nahrung gereichten Muscheln und 4, durch Anwendung von Hilfsmitteln die Schnecken veranlaßt wurden, ihre Beute über dem Sediment anzubohren und zu verzehren.

\section{G. Das Verhalten von Lunatia nitida im Aquarium und Beobachtungen über die Nahrungssuche}

Die meiste Zeit über lebt L. nitida in den Aquarien im Sand verborgen und nur die kreuz- und querlaufenden typischen furchenartigen Spuren auf der Sedimentoberfläche verraten ihr Vorhandensein. Dieses ständige Durchpflügen der etwa $2-3 \mathrm{~cm}$ dicken Sedimentschicht hat zur Folge, daß der Boden reichlich mit Sauerstoff durchmischt wird und der Sand während der Untersuchungszeit nicht erneuert zu werden brauchte. Die Spuren auf dem Sand zeigten auch jedesmal an, wann die Schnecken, nachdem sie mit Muscheln gefüttert wurden und in der Zeit fast an derselben Stelle im Sediment sitend mit dem Bohren und Fressen beschäftigt waren, sämtliche Nahrungstiere überwältigt und aufgezehrt hatten. Sofort waren die frischen Furchen der nahrungssuchend im Boden lebhaft umherkriechenden Tiere $z \mathrm{u}$ beobachten. $\mathrm{Zu}$ den Grableistungen sind die Naticiden befähigt durch den stark schwellbaren und gut entwickelten vorderen Fußabschnitt, das Propodium, das , ,als ein nach oben und hinten auf Kopf und Schale hinaufgeschlagenes, beim Graben schüzendes Schild" (Simrotr) ausgebildet ist.

Setzt aus irgendeinem Grunde einmal die Durchlüftung der Aquarien aus, oder werden Muscheln in ein Becken mit hungernden Schnecken gebracht, oder steigt die Wassertemperatur zu hoch an, dann tauchen die Tiere aus dem Boden auf und kriechen lebhaft auf der Sandoberfläche umher. Gleichmäßig gleitend, als ob die Schnecken dicht über dem Untergrund schweben würden, bewegen sie sich auf dem Sediment mit einer Geschwindigkeit fort, die im Durchschnitt von $2-3 \mathrm{~mm} / \mathrm{sec}$. den von Hirsch bei den Mittelmeerformen beobachteten Werten etwa gleichkommt. Ständig bewegen die Tiere beim Kriechen den Vorderrand des Propodiums unregelmäßig wellenförmig, den Untergrund abtastend. Die bei L. nitida gut erkennbaren pfriemenförmigen und am Grunde abgeplatteten Fühler liegen bei der Fortbewegung über dem Boden etwas nach hinten geneigt mit ihrem Basalteil der Schale auf.

Häufig kriechen die Schnecken an der Aquariumswand nach oben, niemals jedoch soweit, daß sie vollkommen aus dem Wasser sind. Das von Schiemenz ebenfalls bei den Naticiden des Mittelmeeres beschriebene Verhalten, daß die Schnecken aus dem Wasser herauskriechen und so aus dem Aquarium gelangen, wie dies zuweilen bei Nucella lapillus eintritt, konnte bei L. nitida nicht beobachtet werden. Die an der Beckenwand nach oben kriechenden Tiere verhalten an der Wasseroberfläche zuerst kurze Zeit, beugen sich, mit dem Vorderrand des Propodiums die Wạsseroberfläche von unten ein kurzes Stück abtastend, nach hinten, rollen rückwärts ab und fallen dann auf den Boden 
herunter. Häufig kriecht Lunatia umgekehrt an der Wasseroberfläche eine längere Strecke entlang und läßt sich an einem gut erkennbaren Schleimfaden nach unten. In einem Falle kroch ein Tier in dieser Lage 12 Minuten an der Wasseroberfläche.

Werden nach einer Hungerperiode Muscheln in die Becken gebracht, so erscheinen, wie bereits erwähnt, nicht selten schon nach $20-40$ Sekunden die Schnecken auf der Sandoberfläche. Nach einer kurzen Orientierungsdrehung nach dem Auftauchen waren dabei sehr häufig gerichtete Fortbewegungen nach der auf dem Boden liegenden Muschel hin festzustellen. Bei der Nahrungssuche war besonders gut die Funktion, die der vordere Rand des Propodiums als Tastorgan ausübt, zu erkennen. Hatte sich z. B. eine Muschel, noch bevor sie von einer Lunatia aufgespürt wurde, in den Sand eingegraben, und eine Schnecke kam mit dem Vorderfuß an die Stelle, an der die Muschel im Sediment verschwunden war, grub sich die Schnecke sofort ein, und wie häufiges Auswühlen ergab, begann sie sofort nach Erreichen der Muschel mit dem Bohren.

Das Annehmen der Beute ist besonders gut zu beobachten, wenn eine Lunatia eine Muschel auf dem Sand erreicht, bevor diese sich eingraben konnte (Abb. 2 A-F). Sobald die Schnecke mit dem Vorderrand des Propodiums die Muschel berührt hat, ist ein schwaches Zusammenziehen des gesamten Fußes zu erkennen und unmittelbar danach kriecht die Schnecke über die Beute hinweg. Beim Versuch der Lunatia, auf die untere Schalenseite zu gelangen, wird die Muschel angehoben, und durch die das Fortbewegen bedingenden kurzen Wellenbewegungen der Kriechsohle wird die Muschel einige Male im Fuß der Schnecke gedreht (Abb. 2 C). Dabei tastet der Vorderrand des Propodiums jede Stelle der Muschelschale ab. Für den Zweck dieser Drehbewegungen, die bei jeder Schnecke, die eine Muschel über dem Sediment annimmt, zu beobachten ist, könnten folgende Deutungen in Frage kommen: 1. Einschleimen der Beute, 2. Aufsuchen einer Stelle, an der die Schale beschädigt ist, so daß es der Schnecke möglich ist, ohne Bohrakt, den Weichkörper zu verzehren. Bei Versuchen mit lebenden Muscheln als Nahrungstiere, bei denen kleine Schalenstücke ausgebrochen wurden, fraß Lunatia den Weichkörper aus, ohne daß Spuren von Bohrtätigkeit an irgendeiner Stelle der Schale zu erkennen waren. 3. Das Auffinden einer geeigneten Stelle an der Muschelschale, um die Beute in den Sand ziehen zu können, was wohl den Hauptzweck dieser Rollbewegungen bei der über dem Boden angenommenen Beute darzustellen scheint. Bei Schnecken, die Muscheln im Boden anfallen, ist dieses Drehen nicht möglich, dafür umkriecht die Schnecke die Muschel, um die für den Bohrakt günstigste Stellung aufzusuchen.

Nachdem Lunatia eine Muschel über der Sedimentoberfläche einige Male auf der Kriechsohle gedreht hat, heftet sie den hinteren Rand der Kriechsohle, meist an der Kante, zuweilen an der flachen Oberfläche der Muschelschale, mit Hilfe von abgesondertem klebrigem Schleim fest und gräbt sich, entweder bald oder nach längerem Umherkriechen, die Beute hinter sich herziehend, ruckweise in den Sand ein (Abb. 2D-F).

L. nitida hält beim Transport der Beute demnach die Muschel nicht in der Weise fest, wie es Hrrsch für Natica millepunctata und $\mathcal{N}$. hebraea geschildert hat. Nach seinen Beobachtungen kriecht Natica „ein Stück auf die Muschel darauf, krümmt dann den Fuß nach unten und schiebt so den Hinterfuß unter die Beute. Dann wendet sie sich ein wenig mit dem Vorderfuß zur 

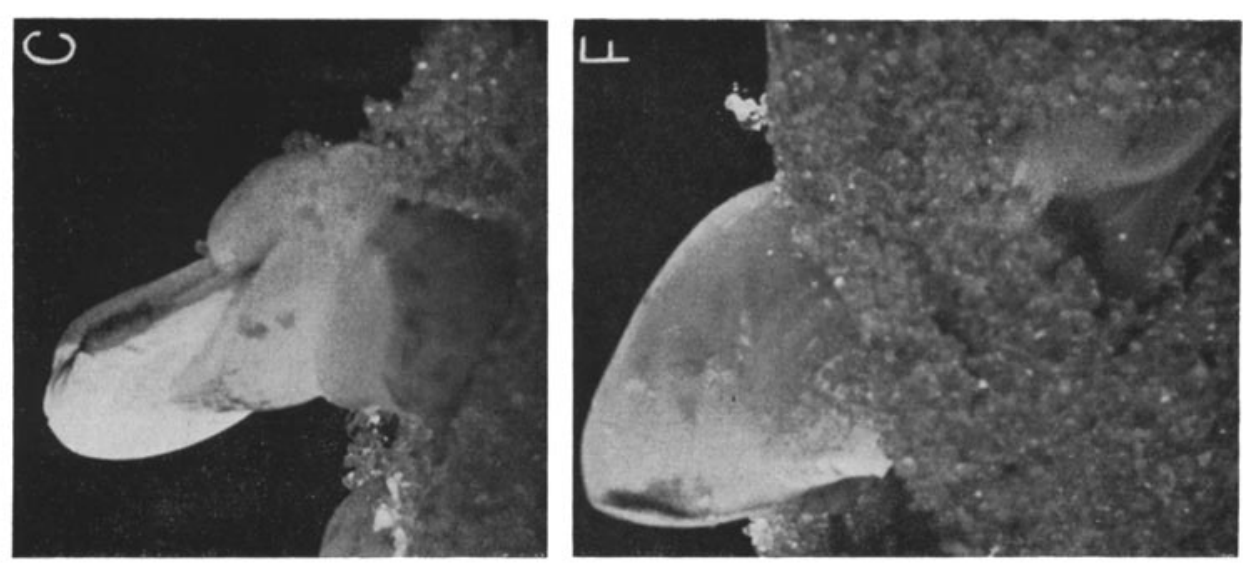

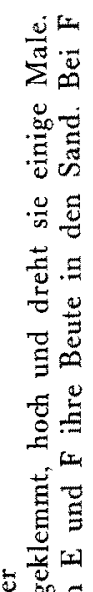
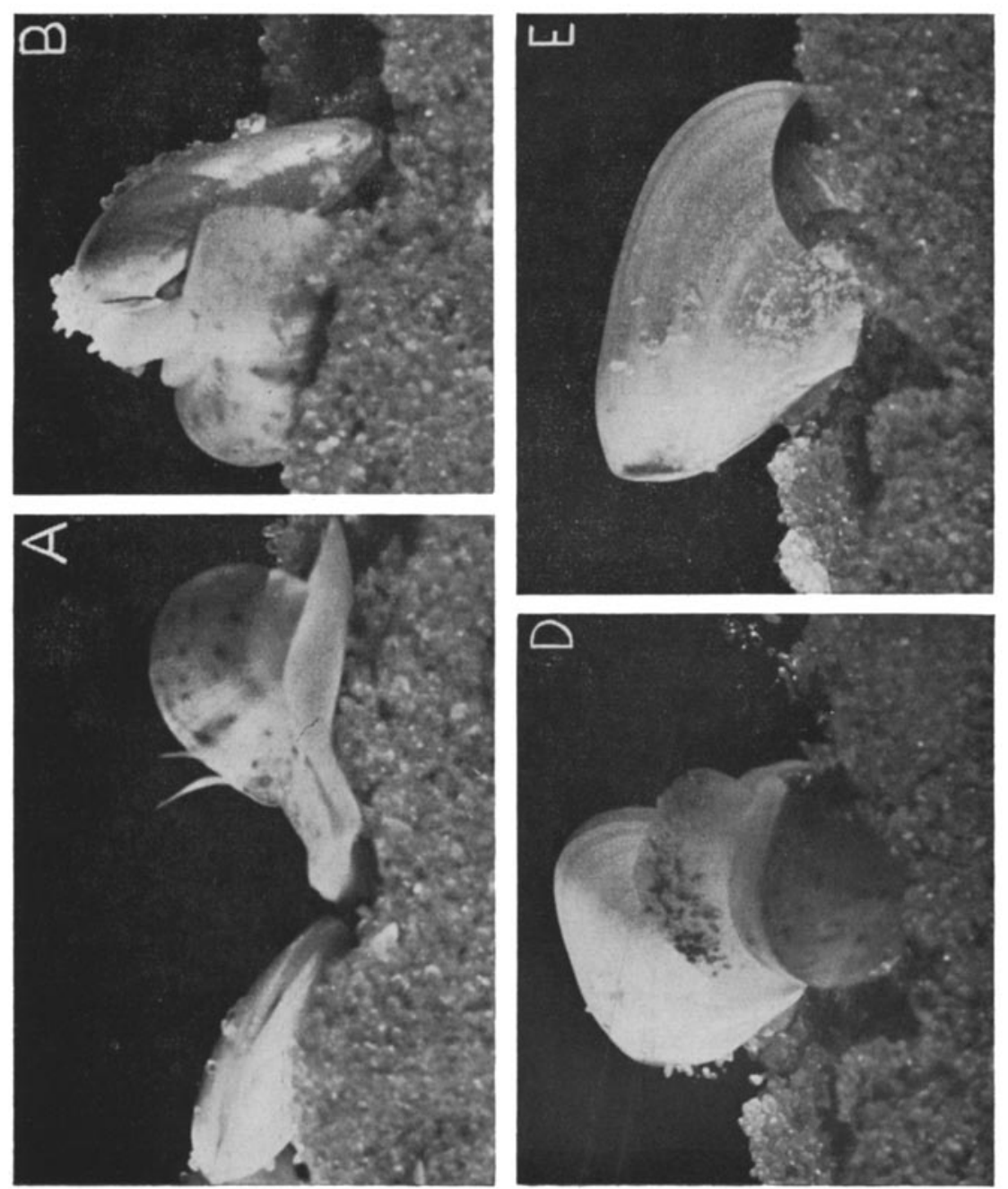

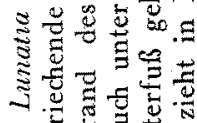

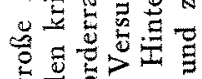

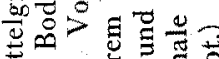

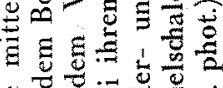

-

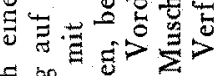

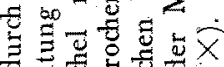

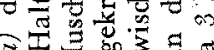

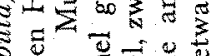

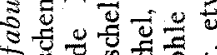

今

.

政

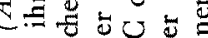

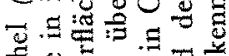

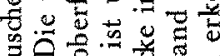

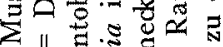

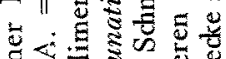

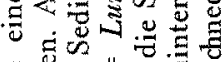

o $\|$

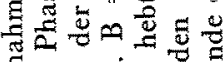

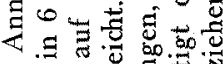

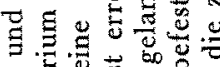

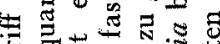

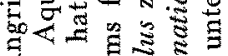

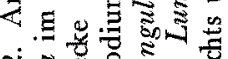

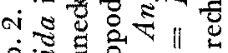

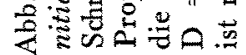


Seite und hat nun zwischen Mittel- und Hinterfuß die Muschel eingeklemmt, wie wir 4 Finger platt gegen die Handfläche legen".

Hat nun Lunatia die Muschel vollkommen in das. Sediment gezogen, dann kriecht sie mit ihrer Beute - im Gegensatz zu dem oben beschriebenen Verhalten beim Anfallen einer Muschel im Boden, wo mit dem Bohren sofort begonnen wird - meist längere Zeit (oft über 1 Stunde) umher, ehe sie den für die Bohrtätigkeit günstigsten Platz gefunden hat. Wird eine mit einer Muschel den Boden durchwühlenden Schnecke von Artgenossen, die ihr die Beute abzunehmen versuchen, gestört, kommt sie auf die Sandoberfläche und kriecht dann mit der angehefteten Muschel (Abb.3) meist an der Glaswand

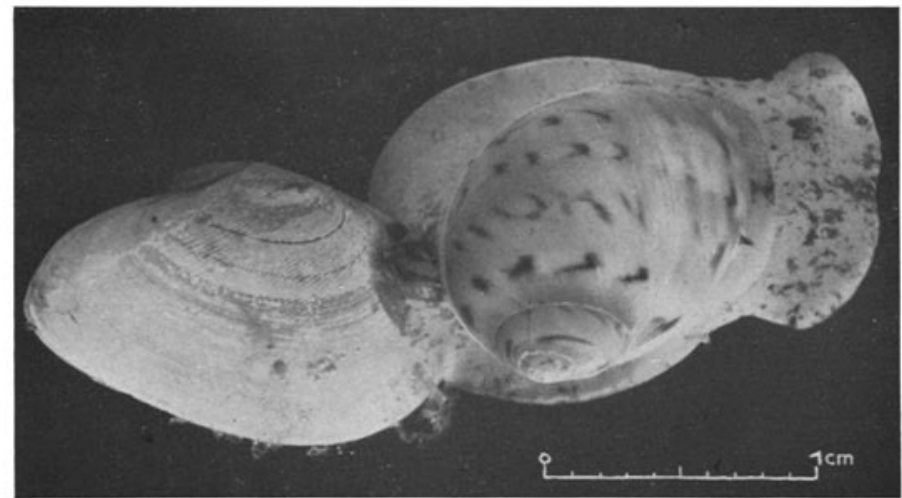

Abb. 3. Lunatia nitida mit einer angehefteten Angulus fabula auf dem sedimentfreien Boden des Aquariums umherkriechend (von oben aufgenommen). (Verf. phot.)

des Beckens bis zur Wasseroberfläche empor. Hierbei läßt sich die Festigkeit, mit der eine Muschel am Fußhinterende angeheftet ist, vor allem bei den die Aquariumswand emporkriechenden Tieren, einmal an dem oft größeren Gewicht der Beute, zum anderen an den starken Bewegungen der Muscheln, die durch heftiges Herausstrecken und Zurückziehen des Fußes sich von den Schnecken loszumachen versuchen, erkennen. Nach wiederholtem Aufsteigen mit der Beute an der Glaswand und Wiederfallenlassen gräbt sich Lunatia dann wieder in das Sediment ein und beginnt, falls sie ungestört bleibt, mit dem Bohrakt.

Die von Hirsch geschilderten Beobachtungen, nach denen die von $\mathcal{N a t i c a}$ angefallenen Mactra durch Sprünge mit Ililfe ihres herausschnellenden Fußes zu entkommen versuchen, konnten bei $L$. nitida durch Versuche mit mittelgroßen Spisula solida bestätigt werden.

\section{Beobachtungen an den von Lunatia im Aquarium gebohrten Löchern}

Mit dem Verschwinden im Sand entzieht sich Lunatia der unmittelbaren Beobachtung der bei der Nahrungsaufnahme wichtigsten Tätigkeiten, des Bohr- und Freßaktes. Es bleibt zunächst nur die Möglichkeit, an Hand der im Aquarium gebohrten Löcher, nach ihrer Lage auf den Muscheln und nach ihrem Aussehen, etwas darüber aussagen zu können, womit die Bohrungen in den Schalen hergestellt werden. 
I. Lage der Bohrlöcher auf den Muschelschalen

Rund 200 Bohrlöcher sind während der Versuche von Lunatia nitida in den Aquarien angefertigt worden. Die Löcher befinden sich hauptsächlich in der Mitte der Schale. an der die Muschel am breitesten ist. Nur bei sehr wenigen liegt das Bohrloch in der Nähe der Schalenkanten. Artgenossen, von denen während der Untersuchungszeit 6 angebohrt wurden, zeigen die Bohrung immer an derselben Stelle und zwar oberhalb der Gehäusemündung (Abb. 5 C). Von den verschiedensten Erklärungen in der Literatur, weshalb bestimmte Stellen auf der Muschelschale von den Schnecken bevorzugt werden, so z. B. um "recht bald zu den Adductoren zu gelangen" (Leidy, zit. nach Schiemenz) oder weil die Schnecken wissen, wo die Gonaden der Beutetiere liegen (Pelseneer), ist wohl die Deutung von C. R. Boettger, der wegen dieser Fragen Versuche mit mediterranen Natica-Arten durchführte, die verständlichste. BoETtger kommt zu dem Ergebnis, daß die Anlage des Bohrloches abhängig ist von der Größe der angegriffenen Muschel, ferner umfassen die Raubschnecken ihre Beute so, damit ,der Bohr- und Freßakt in bequemer Stellung vor sich gehen kann". Die mit $L$. nitida durchgeführten Versuche bestätigen die Ergebnisse Boettger's, worüber bei der Besprechung des Bohrvorganges noch zu berichten sein wird.

Hinsichtlich der Verteilung der Bohrlöcher auf die beiden Seiten der Muschelschalen konnten auffallende Unterschiede bei den im Aquarium angebohrten Muscheln nicht festgestellt werden. Schiemenz führte ein Überwiegen der Bohrlöcher auf der linken Schalenseite darauf zurück, daß Natica die Muscheln meist von vorn angreift, sie umklammert und durch die Rechtswindung des Schneckengehäuses der herausgestreckte Rüssel ebenfalls nach rechts zeigt, wodurch er auf die linke Schalenseite gelangt. Schiemenz ging dabei von der stets gleichen Lage der Muscheln im Sediment aus (mit den ventralen Schalenrändern nach unten). Sehen wir zunächst von der noch zu besprechenden Bohrstellung von Lunatia ab, die sich von der bei Schiemenz für Natica josephinia in einer Kombinationszeichnung dargestellten unterscheidet, ließen Feststellungen zur Lage von Muscheln im Sediment den Schluß $z u$, daß eine Bevorzugung einer bestimmten Schalenseite für den Bohrakt in Frage kommen könnte.

Nach einer Reihe von Beobachtungen bei den Bodengreiferuntersuchungen und bei Aquariumsversuchen (noch unveröffentlicht) liegt Angulus fabula flach im Boden, mit der linken Schalenseite nach unten. Die äußere Form der Schale von Angulus fabula, bei der das Hinterende stets nach der rechten, schräggestreiften Seite hin etwas umgebogen ist - bei den jüngeren Tieren nicht so deutlich wie bei den größeren -, ließ vermuten, daß diese Formveränderung nur durch die langen Siphonen hervorgerufen sein kann, die bei der erwähnten Lage der Muschel im Boden das Schalenhinterende während des Wachstums nach rechts biegen. Bei Macoma baltica und Angulus tenuis - ebenfalls zur Familie der Tellinidae gehörig - ist nach den bisherigen Beobachtungen dieselbe Lage im Sediment, wie sie für Angulus fabula geschildert ist, wahrscheinlich.

So konnte bei der Auswertung der im Aquarium angebohrten Muscheln festgestellt werden, daß nur bei Angulus fabula etwas häufiger die Löcher auf der rechten Seite zu finden sind. Angulus fabula gräbt sich in den Aquarien so weit in den Sand ein, daß sie mit ihrer linken Seite auf den Glasboden zu liegen kommt. Die im Sediment umherkriechenden Raubschnecken treffen bei ihrer Nahrungssuche auf die nach oben liegende Seite der Muschel und beginnen mit dem Bohren. Die rechte Schale wurde bei Angulus fabula häufig dann angebohrt, wenn die Muscheln Zeit hatten, sich weit genug eingraben $z u$ können und von Lunatia in der geschilderten Lage im Boden angegriffen 

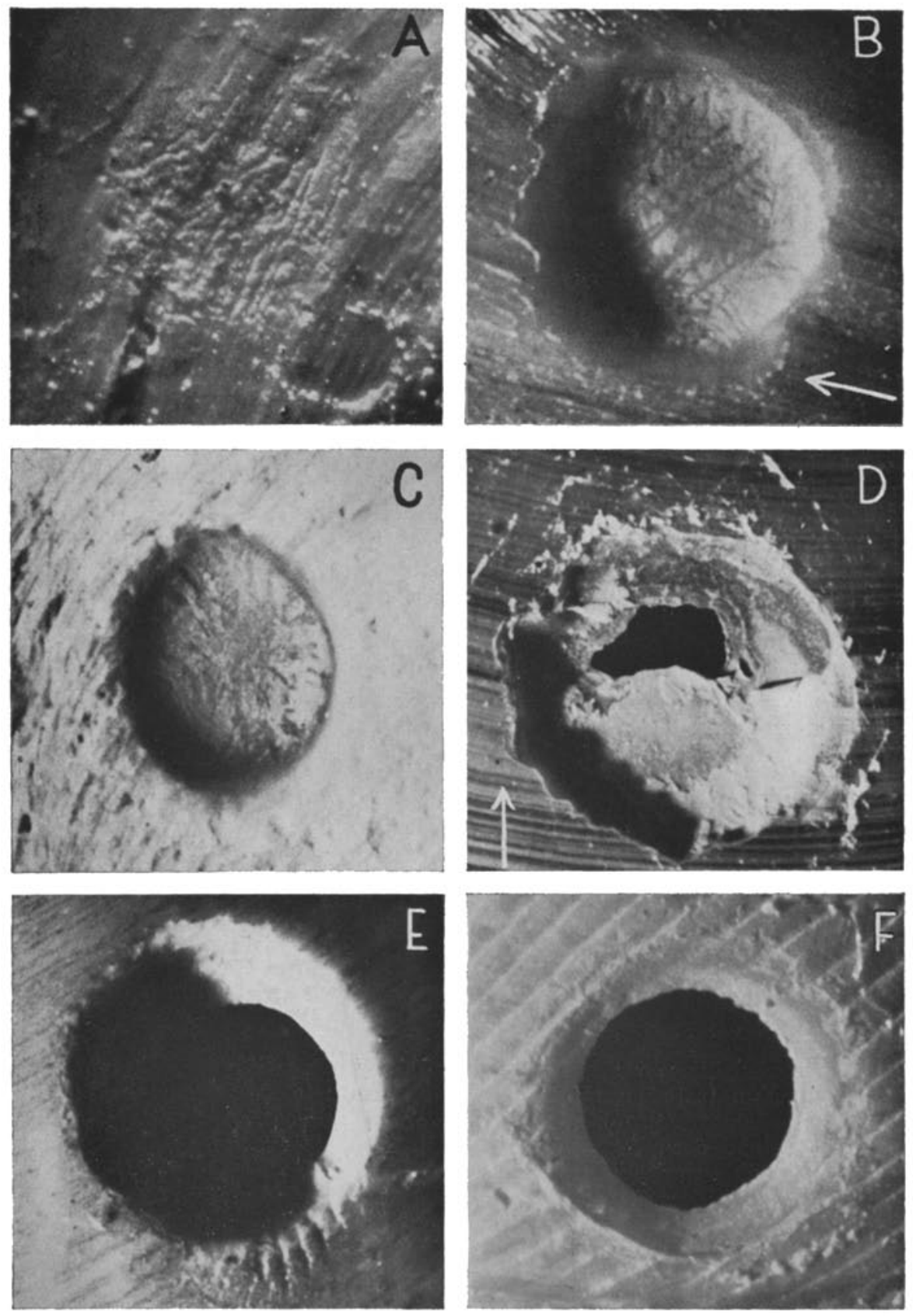

Abb. 4. Zusammenstellung einer Auswahl von Bohrungen, die von Lunatia nitida während der Aquariumsversuche hergestellt worden sind. $A=$ Kratzspuren nach der ersten Raspelperiode (Vergr. $30 \times$ ). $\mathrm{B}=$ Unfertiges Bohrloch mit Raspelspuren in einer großen Spisula subtruncata. Der Pfeil gibt die Stellungsrichtung an, mit der die Schnecke während des Bohraktes auf der Muschel saß (Vergr. 32 X). $\mathrm{C}=$ Radiäre Raspelspuren in einer unfertigen Bohrung in einer Macoma baltica (Vergr. $28 \times) . D=$ Nierenförmiger Durdbruch in einem Bohrloch auf Angulus tenuis (Pfeil wie in B) (Vergr. 30 $X$ ). E = Fertiges Bohrloch in der Schale einer mittelgroßen Spisula subtruncata mit Kratzspuren am äußeren Bohrlochrand (Vergr. $35 \times$ ). F = Fertige Bohrung bei Angulus fabula (Vergr. 29X). (Verf. phot.) 
wurden. Erfolgte das Annehmen einer Muschel während sie sich in den Sand eingrub oder noch auf der Bodenoberfläche lag, legte sich Lunatia die Beute zurecht, und es hing dann vom Zufall ab, auf welcher Schalenseite Angulus fabula angebohrt wurde.

\section{Die Form und Beschaffenheit der Bohrlöcher}

Wieweit läßt sich nun auf Grund der genauen Untersuchung der einzelnen Bohrlöcher, die von L. nitida während der Aquariumsversuche hergestellt wurden (Abb. 4 A-F), etwas über die Bohrweise aussagen?

1. Fertige Bohrungen.

Die Kehlung in den Wänden der konischen Bohrlöcher ist bei den dickeren Schalen deutlicher zu erkennen. Während bei dünnschaligen Muscheln zuweilen ovale Bohrungen festgestellt werden können, sind die Löcher bei dickeren Schalen meist kreisrund. Die Gehäusehöhen der zu den Versuchen verwandten Lunatia nitida liegen zwischen 4-10 mm. Das kleinste Bohrloch hat einen äußeren Durchmesser von $0,7 \mathrm{~mm}$ und einen inneren von $0,6 \mathrm{~mm}$ bei einer $8 \mathrm{~mm}$ Iangen Angulus fabula. Die Werte für die größte Bohrung betragen 2,2 bzw. 1,7 mm (Angulus fabula $=15,5 \mathrm{~mm}$ lang). Loppens gibt für die Löcher, die von derselben Natica-Art gebohrt wurden, das Verhältnis vom inneren zum äußeren Durchmesser bei dünnschaligen mit $1: 1,5$ und bei dickschaligen mit $1: 2,2$ an. In der folgenden Tabelle sind die entsprechenden Durchschnittswerte für die während der Versuche mit L. nitida als Nahrungstiere verwandten Muschelspezies zusammengestellt, woraus deutlich hervorgeht, daß mit zunehmender Schalendicke bei ungefähr gleichbleibendem äußeren Durchmesser bei den entsprechenden Größen von Lunatia der innere Durchmesser immer kleiner wird.

$\begin{array}{llll}\text { Angulus fabula } & 1: 1,29 & \text { Nucula nucleus } & 1: 1,51 \\ \text { Angulus tenuis } & 1: 1,39 & \text { Spisula solida } & 1: 1,52 \\ \text { Mya arenaria juv. } & 1: 1,40 & \text { Nucula nitida } & 1: 1,59 \\ \text { Abra alba } & 1: 1,44 & \text { Venus gallina } & 1: 1,61 \\ \text { Macoma baltica } & 1: 1,49 & \text { Spisula subtruncata } & 1: 1,70\end{array}$

Diese Unterschiede der inneren und äußeren Durchmesser bei Bohrlöchern in dünn- und dickwandigen Muschelschalen sind wohl darin begründet, daß die für die Bohrtätigkeit aufzubringende Energie der Schnecken und die Gebrauchsfähigkeit der Bohrmittel mit der Länge der benötigten Bohrzeit abnimmt, worauf bei der Darstellung der Bohrtätigkeit und der Morphologie des Rüssels noch näher eingegangen wird. Deutlich ist diese Verminderung der Leistung bei Bohrungen in den dickwandigen Schalen von Aloidis gibba zu erkennen (Abb. 5A und B, ferner Abb. 13 A). Diese Muschelart fällt auf durch den asymmetrischen Bau ihrer Schalen, bei denen die rechte größer ist und über die linke greift. Fast ausschließlich befinden sich die Bohrlöcher auf der größeren rechten Klappe, auf der linken Seite wird Aloidis nur ganz selten angebohrt. Die rechte Schale bietet wegen ihrer Form und Größe den Schnecken eine günstigere Unterlage für die Stellung beim Bohrakt. Sehr häufig sind auf der rechten Schale von Aloidis mehrere unfertige Bohrungen zu finden. Die Schnecke ist während des Bohrens auf eine gelbliche, härtere Schicht ${ }^{2}$ ) gestoßen, die als dünne Lage die Schalen durch-

2) Nach Fischer (1922) liegt die Schicht etwa auf $2 / 3$ der gesamten Schalendicke von außen nach innen und besteht aus Konchiolin oder einem nahe verwandten Stoff. 
zieht, und auf der Schaleninnenseite in einer feinen Linie etwa parallel zum Schalenrand ausstreicht. Die fertigen Bohrlöcher in Aloidis-Schalen zeigen stets die gleiche, jedoch eine von der typischen Lunatia-Bohrung abweichende Form: Die Wandungen der Löcher sind nicht gleichmäßig gekehlt und stehen konisch, sondern besitzen eine Stufe, die dadurch entsteht, daß nach der ersten. bis zur Zwischenschicht durchgeführten Bohrung dann ein kleineres Loch von der harten Schicht nach dem Muschelinneren durch die Schale gebohrt wird.

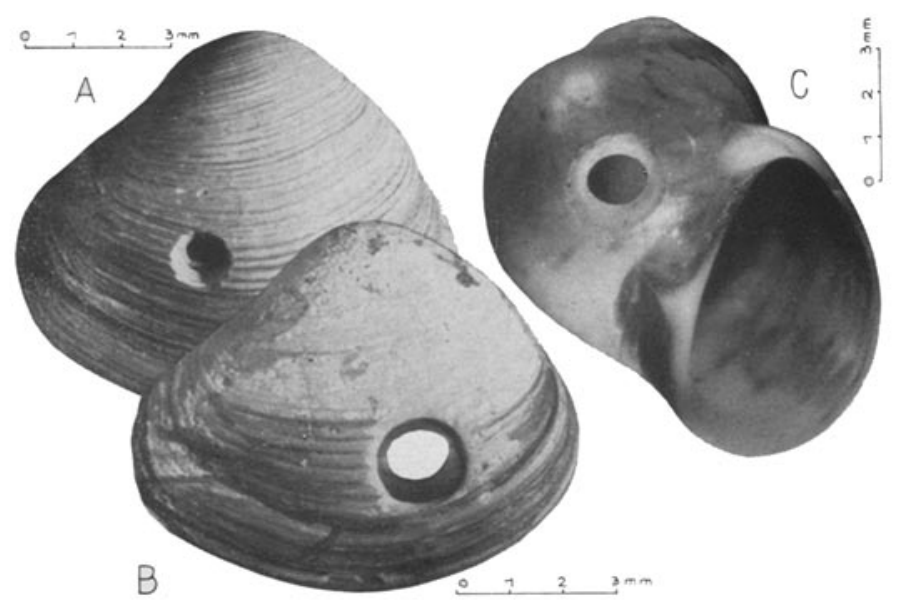

Abb. 5. $\mathrm{A}=$ Unfertiges Bohrloch in der Schale von Aloidis gibba. Die Schnecke ist während des Bohraktes auf die harte $Z$ wischenschicht gestoßen und hat gerade mit deren Durchbohrung begonnen. $\mathrm{B}=$ Fertige Bohrung in Aloidis gibba. Das Loch zeigt die typische Abstufung infolge der Zwischenschicht. $\mathrm{C}=$ Bohrloch im Gehäuse eines Artgenossen, das bei sämtlichen überwältigten Schnecken an derselben Stelle oberhalb der Gehäusemündung liegt. (Verf. phot.)

Bei angebohrten Aloidis-Schalen in Dredsch-oder Bodengreiferfängen könnte vermutet werden, daß die innere zweite Bohrung von einer anderen kleineren Schnecke hergestellt worden ist, nachdem die vorher Bohrende durch irgendwelche Umstände beim Auftreffen auf die härtere Zwischenschicht gestört wurde und die Bohrtätigkeit aufgeben mußte. Die Aquariumsversuche aber zeigten, daß diese Löcher in Aloidis-Schalen nur von einem Tier hergestellt werden.

Betrachten wir die in die Schalen von Aloidis gibba gebohrten Löcher unter dem Gesichtspunkt der beim Bohren von Lunatia angewandten Methode, dann lassen sich hier bereits gegen die „Bohrdrüsen“-Theorie von Schremenz Bedenken äußern̂: Durch eine Säure löst sich die Zwischenschicht nicht auf (Versuche von Fischer mit reiner Salzsäure, eigene Beobachtungen bei Anwendung von Salzsäure und Schwefelsäure). Eine, wie in der Einleitung erwähnt, evtl. in Frage kommende Calcase als Lösungsmittel schaltet aus. Sollte dennoch ein Drüsensekret, dessen chemische Zusammensetzung unbekannt wäre, diese $Z$ wischenschicht auflösen können, dann bleibt unverständlich, weshalb die Schnecke bei Aloidis die tellerförmige Drüse in ihrem Umfang verringert, um die kleinere Offnung durch die harte Schicht zu bohren. Damit wäre ein Beweismittel, mit dem Schiemenz und die Verfechter seiner Annahme ihre Behauptung stütten, da Natica mit chemischen Mitteln bohre, nämlich die Ubereinstimmung der Größen von Bohrdrüse und gebohrten Löchern, hinfällig. 
Einen Hinweis auf die wahrscheinlichere Bohrweise ergaben Beobachtungen an

2. unfertigen Bohrungen

bei anderen Muschelarten. Sehr häufig können bei nicht vollständigen Löchern entweder an den Rändern der äußeren Offnung oder auf dem Grunde der ausgehöhlten Grube Kratsspuren beobachtet werden, die von der Tätigkeit der Radula herrühren. Die Raspelspuren in den Bohrungen der Abb $4 \mathrm{~A}$ bis $C_{1}$ und $E$ sind weder durch Sandkörnchenschliff noch durch unvorsichtige Behandlung der Klappen mit Pinzette, Nadel oder Pinsel entstanden, sondern die Schalen wurden den im Sediment bohrenden Schnecken durch Auswühlen abgenommen oder stammen aus einem Versuchsbecken, das kein Sediment enthielt.

Auf eine Besonderheit, die bei einigen unvollständigen Löchern beobachtet werden kann, sei noch kurz hingewiesen: Mitunter bleibt, etwa in der Mitte der Aushöhlung eine kleine Erhöhung stehen, in unmittelbarer Nähe davon befindet sich häufig schon der erste Durchbruch, der sich nierenförmig um den Mittelpunkt legt (Abb. 4 D). Auf die Entstehungsursache dieser von SchIemenz als "Centralhügel" bezeichneten Bildung wird im Zusammenhang mit der Besprechung des Bohrvorganges noch näher eingegangen.

Die Beobachtungen von einwandfreien Raspelspuren besonders an den nicht fertiggestellten Lunatia-Bohrungen ließen den Schluß zu, daß die „Bohrdrüse" bei dieser Naticide nicht das alleinige Werkzeug im Sinne Schremenz bei der Herstellung der Löcher ist. Nach den auftretenden Kratsspuren muß die Radula beteiligt sein. Auf indirektem Wege wurde nun durch Anwendung von Hilfsmitteln versucht, Lunatia zum Bohren über dem Sediment zu veranlassen, um so die unmittelbare Beobachtung des Bohrvorganges zu ermöglichen.

Es hatte sich bei den Versuchen gezeigt, daß es nicht genügt, die Schnecken nur hungern zu lassen, um sie zum Bohren außerhalb des Sandes zu bringen. Der Hungergrad muß durch eine während der Zeit ausgeübte Tätigkeit der Tiere gesteigert werden. $\mathrm{Zu}$ diesem $\mathrm{Zweck}$ wurden den Schnecken präparierte Muscheln als Futtertiere gegeben. Lebende Muscheln wurden in kochendem Wasser zum Klaffen gebracht, der Weichkörper herausgenommen und die Klappen, entweder leer oder gefüllt, mit einem wasserunlöslichen Klebstoff, einer Kollodiumlösung ( "Uhu“) wieder zusammengeklebt. Bei leeren Muscheln mußte am Vorder- und Hinterende ein kleiner Spalt offen gelassen werden, damit Seewasser eindringen konnte und sie auf der Sandoberfläche liegen blieben.

Im ersten Versuch wurden leere Angulus fabula in die Aquarien getan; am Morgen des darauffolgenden Tages konnten auf den Klappen unfertige Bohrlöcher festgestellt werden. Bei weiteren Versuchen wurden die Schalen mit Gips und mit Knetmasse angefüllt. Bei zwei mit Knetmasse präparierten Doppelklappen wurde zwischen der Knetmasse und Schaleninnenseiten je ein nach der Größe der Schalen zurechtgeschnittenes Stückchen blaues Lackmuspapier gelegt.

Sämtliche Schalen wurden von den Schnecken angebohrt, eine Muschel (mit Knetmasse-Füllung und Lackmuspapier) hatte auf einer Seite zwei Löcher. Lunatia bohrte bei allen Löchern tief in die Füllmasse hinein. Die Ränder der Löcher im Lackmuspapier zeigten keine Rotfärbung, die Kontrolle mit 
sehr stark verdünnter Schwefelsäure ergab, daß das Lackmuspapier die Reagenzfähigkeit behalten hatte.

Während dieser Versuche wurde in ein Aquarium eine große Spisula subtruncata gesetst, die sich nicht ganz in das Sediment eingraben konnte und etwa zur Hälfte aus dem Sand herausragte. Hierbei war die Möglichkeit gegeben, daß die Spisula von einer der mit präparierten Muscheln gefütterten Schnecken evtl. an einem außerhalb des Sediments liegenden Schalenteil angefallen und angebohrt wird. Der Erfolg zeigte sich, als nach etwa 4 Wochen eine Lunatia in der typischen Stellung über dem Boden bohrend an der Spisula beobachtet werden konnte, wie die Abb. 6 A zeigt, die kurz nach dem Ansetzen der Schnecke aufgenommen wurde. Dadurch, daß die Spisula dicht an der Glaswand des Aquariums saß, konnte die Bohrtätigkeit unter dem Binokular verfolgt werden. Die Schnecke hörte nach einigen Stunden zu bohren auf, nachdem nur eine flache, runde Grube ausgehöhlt wurde, an deren Grunde aber sehr deutliche Raspelspuren zu erkennen waren (Abb. 4 B).

\section{E. Direkte Beobachtung des Bohrvorganges}

Für weitere Versuche, die zur unmittelbaren Beobachtung des Bohr-. und Freßaktes führten, ist folgende Methode angewandt worden: Die stark hungernden Schnecken wurden in ein kleines, flacheres Becken gesettt, in dem sich eine etwa $1 \mathrm{~cm}$ dicke Sedimentschicht befand. Wegen der geringen Größse des Versuchsaquariums wurde ständig während der Beobachtungen frisches Seewasser hindurchgeleitet. Das Becken ist so aufgestellt worden, daß dessen Boden mit Hilfe eines um $45^{0}$ geneigten Spiegels stets von unten mit beobachtet werden konnte. In das Versuchsbecken wurden meist nur 1 bis 2 Schnecken eingesetzt. Nach etwa einstündiger Eingewöhnung bekam jede Schnecke je eine lebende Muschel (hauptsächlich Angulus fabula, gelegentlich Angulus tenuis, Macoma baltica sowie Spisula subtruncata und Sp. solida). Da die Schnecken fast immer auf der Oberfläche des Bodens umherkrochen, wurden die Muscheln in unmittelbare Nähe der Schnecken gelegt. Die Annahme der Beute erfolgte in den meisten Fällen sofort. Nach einigen Drehbewegungen (s. S. 6) verschwand Lunatia mit der am Hinterende des Fußes festgehefteten Muschel im Boden. Oft kroch die Schnecke, die Beute hinter sich herziehènd, in der dünnen Sandschicht umher, ehe sie mit der Bohrtätigkeit begann, was sich mit Hilfe des Spiegels sehr gut beobachten ließ. Auf diese Weise konnte der Zeitpunkt des Bohrbeginns ziemlich genau festgestellt werden. Hatte Lunatia mit dem Bohren angefangen, wurde sie etwa 20 bis 30 Minuten ungestört gelassen. Dann ist mit einem dünneren Schlauch vorsichtig das Sediment um die bohrende Schnecke abgesogen worden, so daß Schnecke und Beute frei auf dem Glasboden des Beckens lagen. Nur ganz selten ließen sich die Schnecken dadurch stören und zogen mit ihrem Opfer in das umliegende Sediment, um eine andere Stelle aufzusuchen. Einige Tiere konnten in Bohrstellung aus dem Sand gewühlt werden, sie blieben sitzen und bohrten weiter. Die Sedimentschicht wurde während der Versuche immer dünner gewählt, was schließlich dazu führte, daß Lunatia die Muscheln auf dem reinen Glasboden des Beckens anfiel, anbohrte und den Weichkörper ausfraß. Die Tiere ließen sich bei ihrer Bohrtätigkeit auch nicht stören, wenn sie an die vordere Beckenwand geschoben und für die günstigste Beobachtungsmöglichkeit zurechtgelegt wurden. Mit dieser Methode konnten insgesamt 12 Schnecken (davon 4 in Abb. $6 \mathrm{~A}$ bis D) beim Bohren einwandfrei beobachtet werden. 

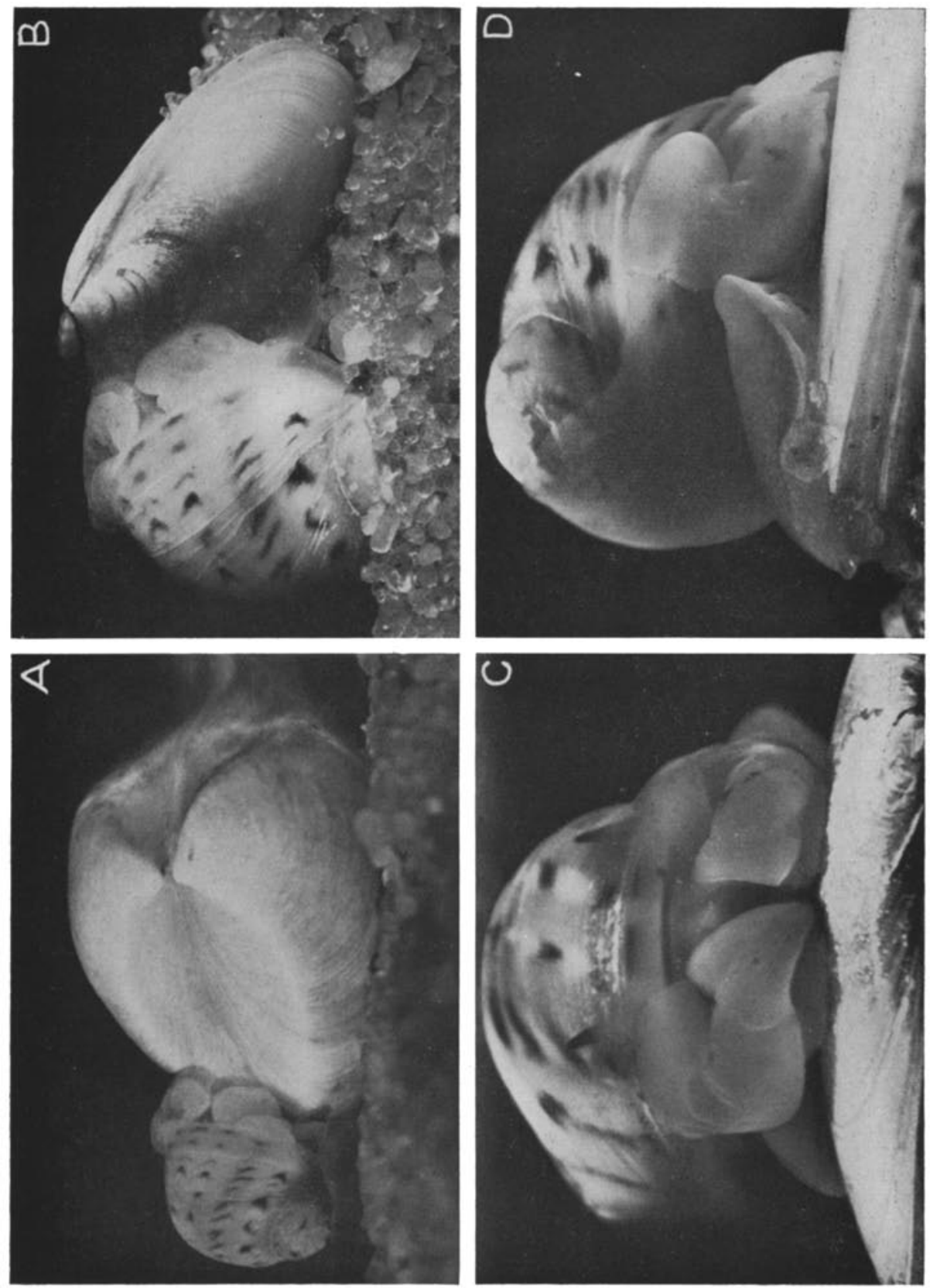

Abb. 6. Aufnahmen von Lunatia nitida, die im Aquarium außerhalb des Sediments Muscheln anbohrten. $\mathrm{A}=$ Erstmalig gelungene direkte Beobachtung des Bohrvorganges an einer Lunatia, die auf einer großen Sprisula subtruncata sitzt (Vergr. 2,5 $\times$ ). B Lunatia nitida eine Angulus fabula anbohrend. Die Schnecke ist mit ihrer Beute für die photographische Aufnahme zurechtgelegt worden, um den ausgestreckten, zwischen den Lappen des Propodiums liegenden Rüssel mit dem nur von oben möglichen Lichteinfall besser auszuleuchten. Am Rüssel ist der schwach durchschimmernde Osophaguseingang zu erkennen (Vergr, 4,5 $X$ ). $\mathrm{C}=$ Lunatia in Bohrstellung (von vorn) auf einer Angulus tenuis, im sedimentfreien Aquarium (Vergr. $7 \times$ ). $\mathrm{D}=$ Lunatia auf einer Angulus tenuis, in Bohrstellung von der Seite. Deutlich sind der vordere und hintere Fußabschnitt und der große, aufquellende und das Gehäuse umhüllende Lappen des Fußes zu erkennen (Vergr. $7 \times$ ). (Verf. phot.) 


\section{Die Herstellung der Bohrlöcher}

Wenn Lunatia eine Muschel angenommen und in das Sediment gezogen hat, kriecht sie so auf eine der Schalenseiten, daß der vordere Rand des Propodiums die in der Kriechrichtung liegende Schalenkante der Muschel erreicht. Bedeckt bei kleineren Beutetieren die Kriechsohle eine Schalenseite nicht ganz, so wird das Hinterende des Schneckenfußes um die Schalenkante nach der anderen Seite umgeschlagen. In dieser Stellung zieht Lunatia den Mittelteil des Propodiums zurück und streckt gleichzeitig den Rüssel aus, der mit seinem Vorderende, der Mundöffnung, um die Länge des ausgestreckten Propodiums vom Schalenrand nach der Mitte zu auf die Muschelschale trifft. Dadurch wird die häufigste Lage der Bohrungen in Mittelfeld der Muscheln verständlich. Durch das Einziehen des mittleren Teils des vorderen Fußabschnitts bilden sich zwei Lappen, die sich um den ausgestülpten Rüssel legen. Sobald das Vorderende des Rüssels auf die Muschelschale stößt, beginnt sofort die Tätigkeit der Radula, die bereits nach den ersten Schabebewegungen deutliche Spuren auf der Muschelschale hinterläßt (Abb. 4 A). Der gesamte Bohrvorgang ist ein periodischer Wechsel von Raspelschlägen und dazwischen liegenden Ruhepausen. Die Schnecken bleiben während des Bohraktes an derselben Stelle sitzen, sie führen keine drehenden Bewegungen aus, mit denen die Entstehung der kreisrunden Löcher erklärt werden könnte. Bevor die Schnecke mit einer Raspelperiode beginnt, dreht sie den Rüssel um dessen Längsachse etwa $90^{\circ}$ nach rechts oder links und fängt sofort an zu schaben. Nach 18 bis 20 Kratgbewegungen tritt Ruhe ein. Eine Raspelperiode dauert 25 bis 30 sec. Während der einzelnen Schabebewegungen wird der Rüssel langsam in die normale Lage zurückgedreht, die Mundöffnung zeigt jeţt wieder in die Längsrichtung der Schnecke (Abb. 7). Von der 10. bis 12. Raspelbewegung an läßt sich deutlich das von der Radula abgeschabte, durch die dünne Rüsselwandung durchscheinende und an den Eingang des Ösophagus transportierte weiße Schalenmaterial erkennen. In der jeder Raspelperiode folgenden Ruhepause, die am Beginn des Bohrens 2 bis 3 Minuten, nach 5 bis 6 Raspelperioden fast genau 5 Minuten dauert, wird der Rüssel von der Unterlage abgehoben und die Mundöffnung dabei etwas nach vorn oben verlagert. Dadurch kommt das tellerförmige Organ ("Bohrdrüse") in die Nähe der Bohrstelle zu liegen. Das Ende einer Ruhepause ist stets daran zu erkennen, daß Lunatia, bevor sie zu raspeln beginnt, den vorderen und hinteren Teil des Fußes kräftig gegen die Unterlage stemmt und den Mittelteil der Kriechsohle etwas hebt. Diese vor jeder Raspelperiode zu beobachtende Bewegung kann wohl nur damit erklärt werden, daß durch den unter der Mitte des Fußes zwischen Muschelschale und Kriedsohle entstehenden Sog die Schnecke fest an die Muschel angedrückt wird. Damit wird auch verständlich, daß Lunatia Muscheln mit stark skulptierten Schalen (wie Venus gallina und Cardium edule, s. S. 3), weniger anfällt, da sie sich während des Bohraktes nicht so an die Unterlage festheften kann, wie dies bei einer glatten Schalenoberfläche möglich ist. Durch das Drehen des Rüssels vor der Raspelperiode um etwa $90^{\circ}$ nach rechts oder links und das Zurückdrehen in die Ruhelage während des Schabens wird das kreisrunde Bohrloch sektorenweise von der Radula ausgehöhlt. Wie die schematische Darstellung (Abb. 7) zeigt, raspelt die Radula nach je einer Links- oder Rechtsdrehung des Rüssels in Richtung auf den vorderen Kreisbogen der Bohrlochwandung. Der hintere Halbkreis der Bohrung entsteht dadurch, daß die Schnecke ihren 
Rüssel wieder nach links oder rechts dreht, ihn dann aber nach der entgegengeseften Seite biegt. Jegt schabt die Radula vom Rand der äußeren Lochöffnung über die Wandung nach der Bohrlochmitte hin. Die Reihenfolge

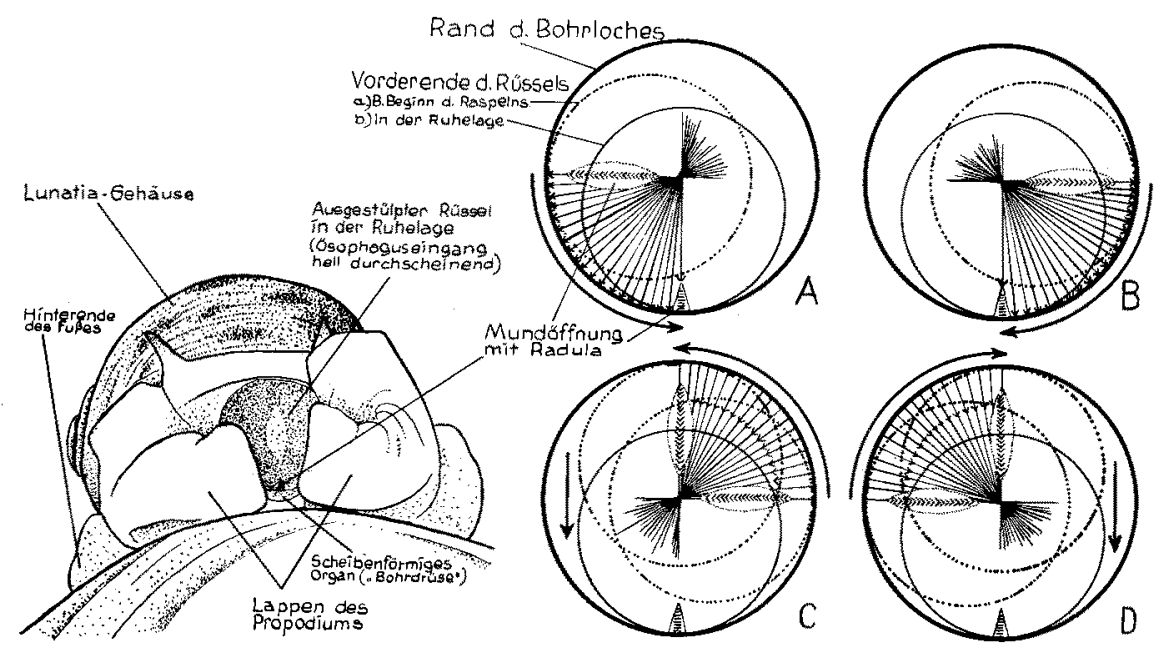

Abb. 7. Links: Lunatia nitida in Bohrstellung auf einer Muschel sitzend, Vorderansicht (nach einer Photographie gezeichnet, etwas schem., Vergr. $4 \times$ ). Rechts: Schematische Darstellung der sektorenweisen Aushöhlung des Bohrloches mit Hilfe der Radula. Die radialen Strahlenbündel geben die Richtung der 18-20 Raspelschläge an, die Winkelleisten an der Mundöffnung zeigen in die Arbeitsrichtung der Radula. $\mathrm{A}=$ Die Schnecke hat den Rüssel um etwa $90^{\circ}$ nach rechts gedreht. Während des Raspelns dreht sie den Rüssel wieder bis zur Ruhelage. $\mathrm{B}=$ Rüssel nach links gedreht, sonst wie bei $\mathrm{A} . \mathrm{C}=$ Der Rüssel ist nach rechts gedreht und dann auf die linke Seite verlagert. Das Raspeln erfolgt nach dem Hinterrand des Bohrloches zu, nach Beendigung der Schabetätigkeit wird der Rüssel nach vorn in dic Ruhelage gebracht.

$\mathrm{D}=$ Rüssel nach links gedreht und auf die rechte Seite verlagert, sonst wie bei $\mathrm{C}$.

der Drehungen des Rüssels wird von der Schnecke während des Bohraktes fast genau eingehalten, wobei die Radula abwechselnd links vorn, rechts vorn, links hinten, rechts hinten arbeitet. Das in Abb. 7 wiedergegebene Schema kann selbstverständlich nicht so aufgefaßt werden, daß die Schnecken während der einzelnen Raspelperioden mit der Radula Schabebewegungen ausführen, deren Spuren Strahlenbündel mit dem Schnittpunkt in der Bohrlochmitte ergeben, sondern die Darstellung soll die Arbeitsrichtungen der Radula nach jeder Rüsseldrehung zeigen und so das Zustandekommen der runden Bohrlöcher veranschaulichen. Wie die Kraţspuren in unvollständigen Bohrungen (z. B. Abb. 4B) zeigen, führt die Radula Raspelschläge aus, die über das ganze Bohrloch reichen, was besonders bei Muscheln mit dickeren Schalen zu beobachten war. Dabei werden während einer Raspelperiode die gegenüberliegenden Teile der vorderen und hinteren Bohrlochwandung zugleich abgeschabt. Bei dünnschaligen Muscheln werden von denselben Schnecken häufig die Bohrungen größer angelegt. Die Aushöhlung erfolgt sektorenweise und führt meist zu der bereits erwähnten Bildung des "Centralhügels" und zum nierenförmigen Durchbruch, der stets im vorderen Teil des Bohrloches liegt, was sich durch die unmittelbare Beobachtung, bei der die Stellung der bohrenden Schnecke auf der Muschel sichtbar ist, einwandfrei feststellen ließ. Bei den größer angelegten Bohrlöchern wird infolge der sektorenweisen Aushöhlung der Mittelteil der Bohrung häufiger ausgespart, es bleibt dann eine kleine Er-

2 Meeresuntersuchungen Bd. V. H. 1 
höhung stehen. Die Entstehung des nierenförmigen ersten Durchbruchs vor dem "Centralhügel" erklärt sich daraus, daß die Radula beim Anseten am wirksamsten schabt, was nach der Schilderung der sektorenweisen Aushöhlung auf dem Grunde der Bohrung im Bereich vor der Mitte der Fall ist. Schiemenz führte die Bildung des "Centralhügels" auf eine zuweilen auftretende Einsenkung in der Mitte der "Bohrdrüse" zurück.

Wird eine Schnecke während des Bohrens durch Anstoßen mit einem Glasstab gestört, zieht sie sofort ihren Rüssel zurück, bei einer kurzen Störung bleibt die Mitte des Vorderfußes eingezogen. Tritt nach einer kurzen einmaligen Störung wieder Ruhe ein, streckt Lunatia den Rüssel aus, und sobald die Mundöffnung die Bohrstelle berührt, beginnt sie mit den Raspelbewegungen. Dauern die Störungen längere Zeit an, dann schiebt die Schnecke nach dem Einziehen ihres Rüssels das eingefaltete Propodium nach vorn in die normale Lage und bedeckt so, etwa mit der Mitte der Kriechsohle, die Bohrstelle. Tritt jetzt Ruhe ein, beginnt sie die Bohrstelle zu suchen, indem der Vorderfuß langsam zurückgezogen wird, bis der ständig tastende Propodiumvorderrand die Bohrung erreicht hat. Sofort streckt Lunatia den Rüssel aus, und die Radula beginnt unmittelbar danach mit dem Raspeln. Werden die Schnecken von der Muschel gewaltsam entfernt, dann ziehen sie sich vollkommen in ihr Gehäuse zurück und bleiben meist längere Zeit neben der Beute liegen. Nach dem Ausstrecken wird die Muschel sofort wieder angenommen und abtransportiert. Keine der so gestörten Schnecken fing nach Annahme der Beute sofort wieder mit dem Bohren an.

Schnecken, die sich einem bohrenden Artgenossen nähern, werden dadurch abgewehrt, daß Lunatia den Rüssel für eine ganz kurze Zeit von der Bohrstelle abhebt und nach dem Angreifer hin biegt. Beim Auftreffen der Mundöffnung auf den Körper der störenden Schnecke führt die Radula einen Raspelschlag aus, bei dem die gepreizten Randzähne der Zunge gut sichtbar sind. Die Wirksamkeit der Abwehr ist eindeutig an dem plötlichen $\mathrm{Zu}-$ sammenzucken der Schnecken zu erkennen, die ihren Angriff meist nicht mehr wiederholen, sondern davonkriechen.

\section{Dauer des Bohraktes}

$\mathrm{Da}$ als Nahrungstiere für Lunatia während der Versuche hauptsächlich Angulus fabula benutgt wurde, konnten die Bohrzeiten besonders für diese Muschelart genau ermittelt werden. Der Beginn des Bohrens wurde in der oben geschilderten Weise festgestellt, das Eindringen des Rüssels am Ende der Bohrtätigkeit war bei den über dem Sediment angebohrten Muscheln an dem ruckartigen Ausstoßen des Grabfußes und an dem plötzlichen Zurückziehen der Siphonen zu erkennen. Lunatia mit einer durchschnittlichen Schalenhöhe von $8 \mathrm{~mm}$ braucht für das Abraspeln von $0,1 \mathrm{~mm}$ Schalendicke be Angulus fabula rund 4 Stunden. Entsprechende Werte ergaben Beobachtungen bei den anderen Muschelspecies, die als Nahrungstiere während der Versuche verwandt wurden, außer Aloidis gibba. Bei Aloidis gibba konnte die Bohrzeit wegen der harten Zwischenschicht nicht festgestellt werden. (Als Vergleich die Bohrzeit bei Nucella lapillus nach eigenen Aquariumsbeobachtungen: $0,1 \mathrm{~mm}$ Schalendicke bei Mytilus edulis in etwa 7 Stunden) $\left.)^{3}\right)$.

3) PIÉRon (1933) gibt an, daß Murex erinaceus für das Bohren von $0,1 \mathrm{~mm}$ Schalendicke benötigt: bei Cardium 7,5 bzw. 8,3 Stunden, bei Tapes 8,3 bzw. 13,3 Stunden. 


\section{Der Freßakt}

Wenn Lunatia die Muschelschale durchbohrt hat, beginnt sie sofort mit dem Ausfressen des Weichkörpers. Ständige Schluckbewegungen sind am Osophaguseingang noch zu erkennen, solange die am Bohrloch liegenden Weichteile aufgezehrt werden. Bald dringt der Rüssel aber immer tiefer in das Muschelinnere ein, die beiden Lappen des Propodiums legen sich vorn dicht zusammen, so daß der Rüssel nach vorn vollkommen abgeschlossen ist. In dieser Stellung sitzt die Schnecke bis der Weichkörper ausgefressen ist. Mit Hilfe sehr starker Durchleuchtung ließ sich bei dünnen Schalen der im Innern der Muschel arbeitende, schwach rötlich durchschimmernde Rüssel erkennen, der bei 8 bis $10 \mathrm{~mm}$ großen Schnecken bis $10 \mathrm{~mm}$ vom Bohrloch entfernt in die Muschel eindringen kann. Zum Aufzehren des Weichkörpers einer Angulus fabula (15 mm lang, $9 \mathrm{~mm}$ hoch) benötigt eine $8 \mathrm{~mm}$ große Lunatia $=2^{1 / 2}$ Tage. In dieser Zeit nimmt die Schnecke eine etwa ihrer Größe entsprechende Menge als Nahrung auf. (Das Rohgewicht des Weichkörpers einer lebenden Lunatia nitida mit einer Schalenhöhe von $8 \mathrm{~mm}$ beträgt $0,130 \mathrm{~g}$, das einer $15 \mathrm{~mm}$ langen Angulus fabula $=0,120 \mathrm{~g}$ ). Bei größeren Muscheln verzehrt die Schnecke vom Weichkörper soviel, als sie mit ihrem Rüssel vom Bohrloch aus erreichen kann. Nach Schätzungen beträgt die aufgenommene Menge zuweilen das $1^{1 / 2}$ fache des Weichkörpers der fressenden Lunatia. In jeder Muschel werden von den Schnecken die Schließmuskeln zuleţt angegriffen, was den Tieren die Möglichkeit gibt, den gesamten Weichkörper aus den geschlossenen Schalen herauszuholen, ohne daß diese klaffen, wodurch der Weichkörper sich schneller zersetzen würde und für die nur frisches Muschelfleisch fressenden Schnecken nicht mehr verwertbar wäre.

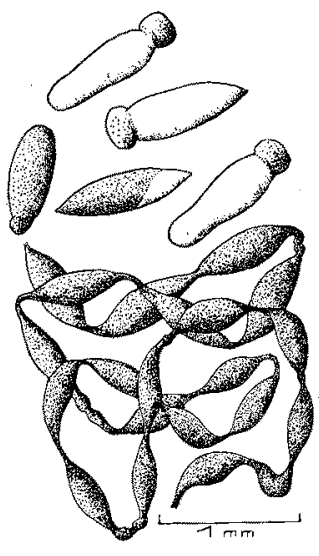

Abb. 8. Kotschnüre, die während des Freßaktes von Lunatia nitida ausgestoßen werden. Oben die einzelnen weißen Kalkspindeln, darunter die zusammenhängenden Glieder der Kotschnüre von schleimiger Konsistenz.

Die Defäkation während des Freßaktes beginnt mit der Ausscheidung von einzelnen weißen Spindeln, die von dem beim Bohren abgeschabten Schalenmaterial stammen. Diesen Spindeln folgen Kotschnüre, die zuerst aus einzelnen, später aus zusammenhängenden, an den Enden zugespitzten Gliedern bestehen. Die Glieder sind etwa $1 \mathrm{~mm}$ lang, in der Mitte ca. 0,2 bis $0,3 \mathrm{~mm}$ dick, schwach gelblich-grün gefärbt und von schleimiger Konsistenz (Abb. 8). Die Anzahl der Kalkspindeln hängt ab von der Dicke der durch- 
bohrten Weichtierschalen. Bei dünnschaligen Muscheln, wie Augulus fabula, Abra alba werden 3 bis 4 , bei dickeren Schalen (Macoma baltica, Spisula u. a.) bis 12 Kalkspindeln ausgeschieden. Ihre durchschnittliche Länge beträgt bei einer 8 bis $10 \mathrm{~mm}$ großen Lunatia 1 bis $1,2 \mathrm{~mm}$, der größte Durchmesser $=0,25$ bis $0,30 \mathrm{~mm}$. Der Vergleich des Rauminhalts der Kalkspindeln mit der Menge des abgeschabten Schalenmaterials bei den einzelnen Muscheln läßt darauf schließen, daß der größte Teil des Kalkes wieder ausgeschieden wird, die genaue Erfassung des Kalkanteils in den Ausscheidungen wird dadurch erschwert, daß die den Kalkspindeln folgenden ersten Glieder der Kotschnüre noch weiße Kalkpartikel enthalten. Daß es sich bei den ausgeschiedenen weißen Spindeln um reine abgeschabte Schalensubstanz handelt, die aus kohlensaurem Kalk besteht, beweist die Behandlung der Spindeln mit Salzsäure. Unter heftigem Aufschäumen werden die Spindeln vollkommen aufgelöst. Damit ist ein weiteres Argument gegen die Bohrdrüsen-Theorie von Schiemenz gegeben: Das von der Radula abgeraspelte Schalenmaterial wird - ohne vorheriges Aufweichen durch ein säurehaltiges Sekret - zum Ósophagus befördert, passiert den Darmtraktus und wird wieder ausgeschieden.

Nach dem Verzehren des Muschelweichkörpers verläßt Lunatia die klaffenden Schalen und kriecht lebhaft im Becken umher. Schnecken, die vorher stark hungerten, griffen häufig unmittelbar danach erneut eine Muschel an, bohrten die Schale durch und fraßen den Weichkörper aus. Die dann angebotenen Muscheln wurden nicht mehr angegriffen. Abgesehen von dem Sättigungsgrad sind die Schnecken wegen der starken Abnützung der Radulazähne (s. S. 25, Abb. 9 und 13) auch nicht mehr imstande, die Schalen durchzubohren. Nur bei einer Lunatia konnte beobachtet werden, daß sie nach zwei Tagen eine dritte Muschel annahm. Wie lange jedoch das Tier für den Bohrund Freßakt benötigte, ließ sich nicht ermitteln, da die Schnecke im Sediment des Hälterungsbeckens saß und nicht gestört wurde.

Allgemein konnte bei den Aquariumsversuchen noch festgestellt werden, daß die Freßlust der Schnecken während der Zeit vom September 1952 bis September 1953 in den Monaten März bis Juni 1953 am größten war.
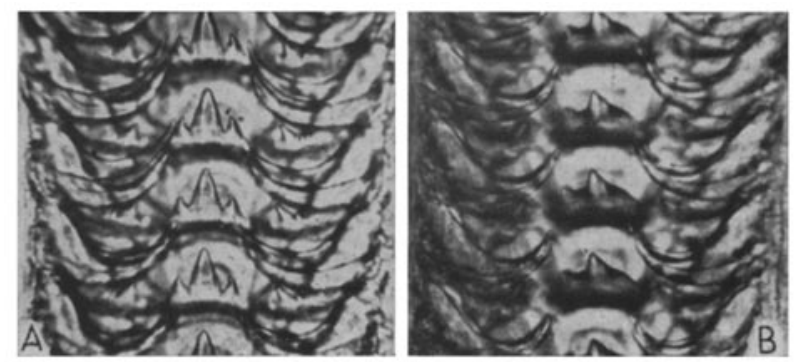

Abb. 9. Mikroaufnahmen von der Radula einer Lunatia, die nach dem Bohr- und Freßakt präpariert wurde. $\mathrm{A}=$ Unverbrauchte Glieder aus dem hinteren Teil der Radula. $\mathrm{B}=$ stark abgenutzte Mittelzähne von Gliedern aus dem wirksamen vorderen Bereich der Radula (Vergr. 8I X). (Verf. phot.)

\section{F. Bau und Funktion des Lunatia-Rüssels}

\section{Der Bau}

Lunatia nitida besitgt, wie alle Naticiden, einen akrembolischen Rüssel, der von der Spite her zurückgezogen wird. Er liegt in eingestülptem Zustand 
(bei einer kriechenden Schnecke) unter der Schale, bedeckt vom Mantel, in der Nähe der vorderen Kante der Gehäusemündung zwischen den beiden Fühlern. Bei verschlossenem Gehäuse wird der Rüssel ein wenig nach hinten verlagert. Ausgestreckt ist der Rüssel gut an dem hellrot gefärbten, durch den Hautmuskelschlauch scheinenden Pharynx zu erkennen. Durch Zertrümmern der Schale von lebenden Schnecken läßt sich der durch die Reizung etwas ausgestülpte Rüssel leicht herauspräparieren.

Die Morphologie des Naticiden-Rüssels in seiner Gesamtheit ist durch die Beschreibungen von Troschel (1856-1866) und Sтмroth im wesentlichen bekannt, es genügt zur Orientierung der Hinweis auf die Abb. 10 A. Notwendig aber für das Verständnis der von den Nabelschnecken vollbrachten Bohrleistungen ist die eingehendere zusammenhängende Darstellung von Bau und Funktion des mit dem Radulamechanismus unmittelbar in Verbindung stehenden Pharynx und seiner Teile.

Der Pharynx (Abb. $10 \mathrm{~A}-\mathrm{C}$ ), der den größten Raum innerhalb der vorderen Rüsselscheide einnimmt, stellt eine schwach abgeflachte, ungefähr herzförmige Kapsel mit stark muskulösen Wänden dar, an der sich deutlich zwei Abschnitte erkennen lassen. Der vordere umschließt die Mundhöhle mit den Kiefern, der hintere den Radulaknorpel (Radulastüte). An der Oberseite des Pharynx mündet, von hinten und unten kommend, der Ósophagus ein. An der Übergangsstelle zur Schlundkopfdecke bildet der Osophagus zwei schwach gebogene leistenartige Verstärkungen, die der oberen Pharynxwand flach aufliegen (Abb.10B) und mit ihren vorderen Enden bis zu den Kiefern reichen. Der so aufgespaltene Osophaguseingang ist mit dem Hautmuskelschlauch des Rüssels durch zahlreiche feine Muskelstränge verbunden, durch welche die Leisten mit ihren Innenkanten gegeneinander bewegt werden und so die vorn beginnenden peristaltischen Bewegungen des Osophagus beim Schluckakt bewirken. Zwischen den Leisten ist eine dünne elastische Membran ausgebildet, die den Osophaguseingang gegen das Rüssellumen abschließt. Dem Osophaguseingang ungefähr gegenüber tritt die Radulascheide aus dem Pharynx, die ein kurzes Stück von dem etwas dahinter ansetyenden starken Rückziehmuskel umschlossen wird, dann nach oben abbiegt und sich hinter dem Schlundkopf in einem Bogen entweder links oder rechts neben Osophagus und Retractor legt.

Die Mundhöhle beginnt hinter der spaltenförmigen, dorso-ventralstehenden Mundöffnung, deren Ränder mit dem Hautmuskelschlauch des Rüssels verwachsen sind. Außen wird die Mundöffnung von einem wulstartigen Polster umgeben, an das sich ventral eine lippenförmige Verstärkung des vorderen Rüsselteils anschließt (Abb. 11). Die kutikulare Auskleidung der Mundhöhle, die sich nach hinten bis zur Radulastütge trichterartig verbreitert, besteht aus zwei Platten (Abb. 12), für die die Bezeichnung „Kiefern“ m. E. nicht ganz zutrifft, da mit diesem Begriff die Vorstellung einer andersartigen Wirkungsweise (z. B. bei Polychäten) verbunden ist. Diese braungelb gefärbten plattenförmigen, nicht planliegenden, sondern der Trichterform der Mundhöhle angepaßten Wandverstärkungen, seţen sich aus einem Mosaik kleiner Stäbchen zusammen, die von Epithelzellen im hinteren Mundhöhlenbereich abgesondert werden. Die Stäbchen sind nach ihrer Entstehungszeit in Reihen angeordnet, die von oben schräg nach vorn unten verlaufen. Die ältesten Stäbchen stehen z. T. frei und bilden die gesägt erscheinenden Vorderränder der Platten, die den Rand der Mundöffnung etwas überragen. Die kutikularen 

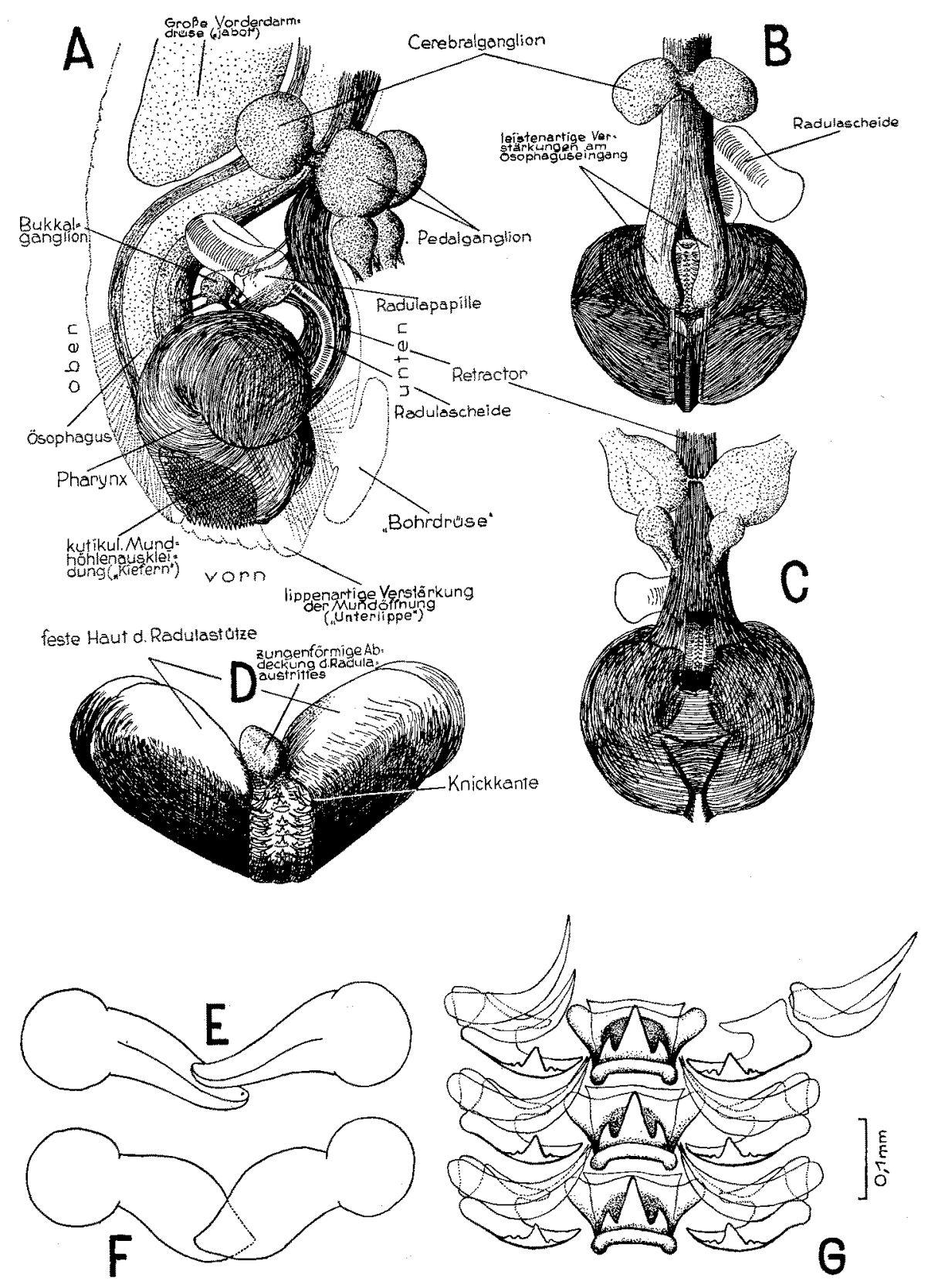

Abb. 10. Bau des Rüssels von Lunatia nitida nach etwas schematisierten Präparationsskizzen (A bis F). Die Ganglien in A-C sind stark schematisiert, auf dem Pharynx ist die Lage der Muskeln durch Schraffur angedeutet. $\mathrm{A}=$ Totalansicht der inneren Rüsselteile; der Hautmuskelschlauch der Rüsselscheide sowie die vom Pharynx und dem Osophaguseingang zur vorderen Rüsselscheidenwand ziehenden Muskeln sind punktiert eingezeichnet. $\mathrm{B}=$ Pharynx und Osophagus von oben, $\mathrm{C}=$ Pharynx von unten, $\mathrm{D}=$ Radulastütze, $\mathrm{E}=$ Zungenknorpel, Vorderansicht, $\mathrm{F}=$ Zungenknorpel, Aufsicht, $\mathrm{G}=3$ Radulaglieder nach einer Mikrophotographie gezeichnet; die Randzähne des obersten Gliedes sind links etwas gehoben, rechts ganz gespreizt (Vergr. A bis C etwa $15 \times, \mathrm{D}$ bis $\mathrm{F}$ etwa $26 \times$ ). 


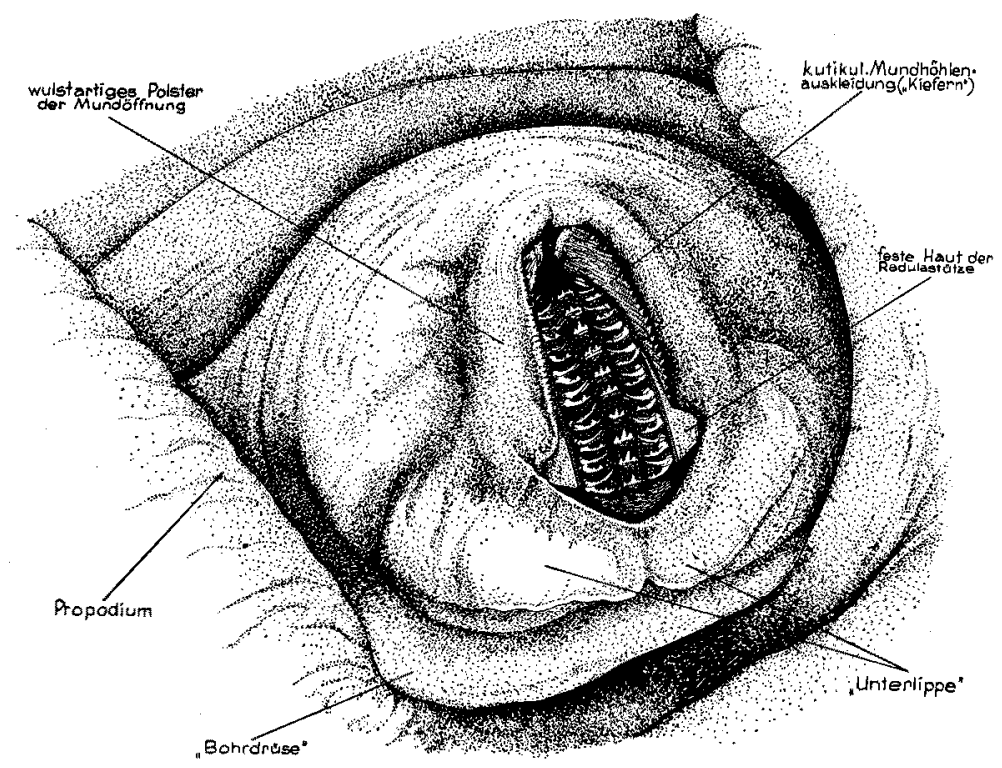

Abb. 11. Vorderer Rüsselteil mit Mundöffnung von Lunatia nitida, gezeidnet nach lebenden Tieren, deren Rüssel durch Zertrümmern der Schale sich etwas ausstreckt. Die Radula ist bei halber Senkung der Radulastütze dargestellt, am oberen Ende des wirksamen Radulaabschnitts ist mit den gespreizten Randzähnen die Knickkante angedeutet (Vergr. $38 \times$ ).
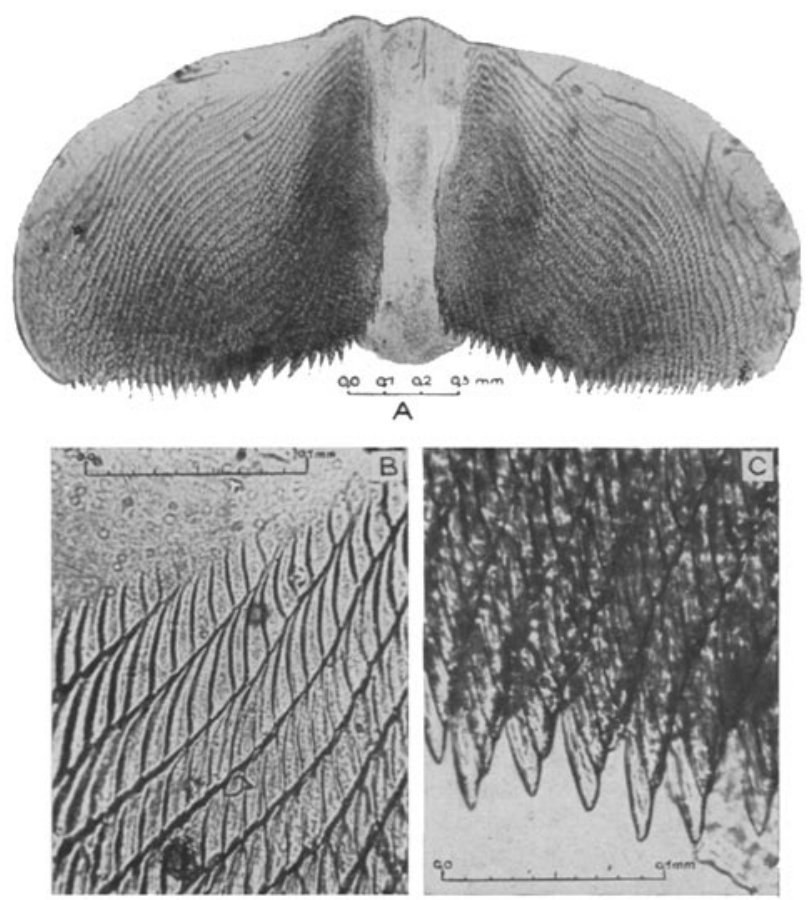

Abb. 12. Kutikulare Mundhöhlenauskleidung („Kiefern“) von Lanatia nitida. A = Total, $\mathrm{B}=$ Bildungszone der Stäbchen im hinteren Abschnitt, $\mathrm{C}=$ Teil des freistehenden gesägten vorderen Randes der Platten bei stärkerer Vergrößerung. (Verf, phot.) 
Wandverstärkungen sind an ihren Oberkanten durch eine dünne elastische Membran verbunden und bilden so die Verlängerung der oberen Decke des Osophaguseinganges bis zur Mundöffnung und könnten schließlich mit einem Gaumen verglichen werden. Die Platten kleiden nach unten bis zu etwa $2 / 3$ die Mundhöhle an den Seiten aus und können mit den Mundhöhlenwandungen bei der Hebung und Senkung der Radulastütge auseinanderklaffen und wieder enger zusammengelegt werden. Sehen wir davon ab, daß die Platten keine „scharfen Schneiden“" besizen, dann ist bei L. nitida auf Grund der in Richtung der Rüsselachse stehenden gesägten Vorderränder der Mundhöhlenplatten ihr scherenartiges Gegeneinanderwirken, das Ankel (1938 b) als eine „Besonderheit der Naticiden" bezeichnet, nicht möglich. Der Zweck der Platten kann demnach nicht die "schneidende Wirkung" sein, sondern eine ziehende und reißende, wodurch das Tier beim Freßakt befähigt wird, Stücke aus dem Weichkörper der Muscheln zwischen den zusammengelegten Plattenvorderrändern festzuhalten und herauszuziehen. Weiterhin stellt die kutikulare Mundhöhlenauskleidung einen Schutz gegen die scharfen Radulazähne und ein für die arbeitende Radulastütze notwendiges Widerlager dar, das in seiner Wirkungsweise mit einem „Bohrfutter" verglichen werden kann. Die Mundhöhlenplatten von Lunatia catena zeigen das gleiche Aussehen wie die für $L$. nitida beschriebenen und sind ebenso in der Mundhöhle angeordnet.

Den Hauptteil des Pharynx bildet der hinter der Mundhöhle liegende Abschnitt, dessen seitliche halbkugelige Ausbuchtungen die im Inneren ansebzende Radulastütze erkennen läßt. In ihrer Gestalt ist die Radulastütge von L. nitida der von ANkEL (1936 b) für die taenioglosse Littorina beschriebenen sehr ähnlich und bildet ebenfalls eine eingewinkelte, „keilförmig zugespitzte Unterlage" für die Radula (Abb. 10 D). Fast die gesamte Oberfläche der Radulastütse ist mit einer festen Haut bedeckt, HuxLEY nennt sie die "elastische Platte", zit. nach Troschel, die zu beiden Seiten der in der Mitte der Stütze liegenden Radulabasalmembran, mit dieser am Rand verwachsen, bis fast zu den Ansatsstellen der Radulastütze an der Pharynxwand reicht. Das Innere der Radulastüte ist hohl. Zwei knorpelige Spangen, nach Troschel. „Zungenknorpel" (Abb. 10 E und F), ragen aus den seitlichen Muskelpolstern frei in den Hohlraum der Radulastütze, wobei die sich überlagernden freistehenden Enden mit ihren Vorderkanten hinter die Basalmembran der Radula zu liegen kommen, die etwa an dieser Stelle in einem scharfen Knick nach unten gebogen ist (nach ANKEL „Knickkante“). Die Knickkante spielt im Radulamechanismus eine entscheidende Rolle, da sie das Spreizen der für das Ergreifen von Nahrungsteilchen wichtigen Randzähne bewirkt. Die typische Spreizzahn-Radula von $L$. nitida besitzt etwa 70 Glieder, die Einzelglieder (Abb. 10 G) bestehen aus einem kräftigen Mittelzahn, zwei Seitenzähnen und je zwei längeren, gebogenen spitzen Randzähnen. Während der Mittelzahn und die Seitenzähne mit ihrer Hauptmasse fest in der Basalmembran verankert sind, sizen die Randzähne nur mit ihrem breiten Ende gelenkartig in der Basalmembran und richten sich an der Knickkante auf.

Für die Bewegung der Radula über die Knickkante bestehen nun zwei Möglichkeiten: Entweder die elastische Platte wird durch Muskelzug abwechselnd nach vorn und hinten gezogen, so daß die Radula über die Knickkante läuft und die Randzähne der einzelnen Glieder nacheinander gespreizt werden (vgl. Ankel, 1936b bei Littorina) oder die elastische Platte mit der Radula liegen fest und die Knickkante der Radulastüze wird verschoben. Im 
Enderfolg sind beide Bewegungen gleich. Verständlicher und einfacher ist allerdings die zweite Möglichkeit, weil bei der ersteren durch die periodischen Bewegungen der Radula über die Radulastütye während der Raspeltätigkeit ein Vor- und Zurückziehen der Radula in deren Scheide außerhalb des Pharynx erfolgt, was zu einer unzweckmäßigen Beanspruchung der Basalmembran im nicht wirksamen Teile der Zunge führen würde.

Bei L. nitida konnte an auspräparierten, überlebend gehaltenen Rüsseln beobachtet werden, dafs die Knickkante durch den Druck der Zungenknorpel nach unten verlagert wird und so die Randzähne der Einzelglieder nacheinander spreizen (Abb. 14). Der wirksame Teil der Radula läßt sich besonders gut an der Abnutsung der Zähne bei Tieren erkennen, die unmittelbar nach dem Bohrakt präpariert werden. Danach verlagert sich die Knickkante auf der Radulastüze durch die Tätigkeit der Zungenknorpel bei jedem Raspelschlag nach oben oder unten um den Abstand von 4-5 Radulagliedern. Die Anzahl der Glieder vom Knidkkantenbereich bis zum Ende der Radula beträgt 9-10.

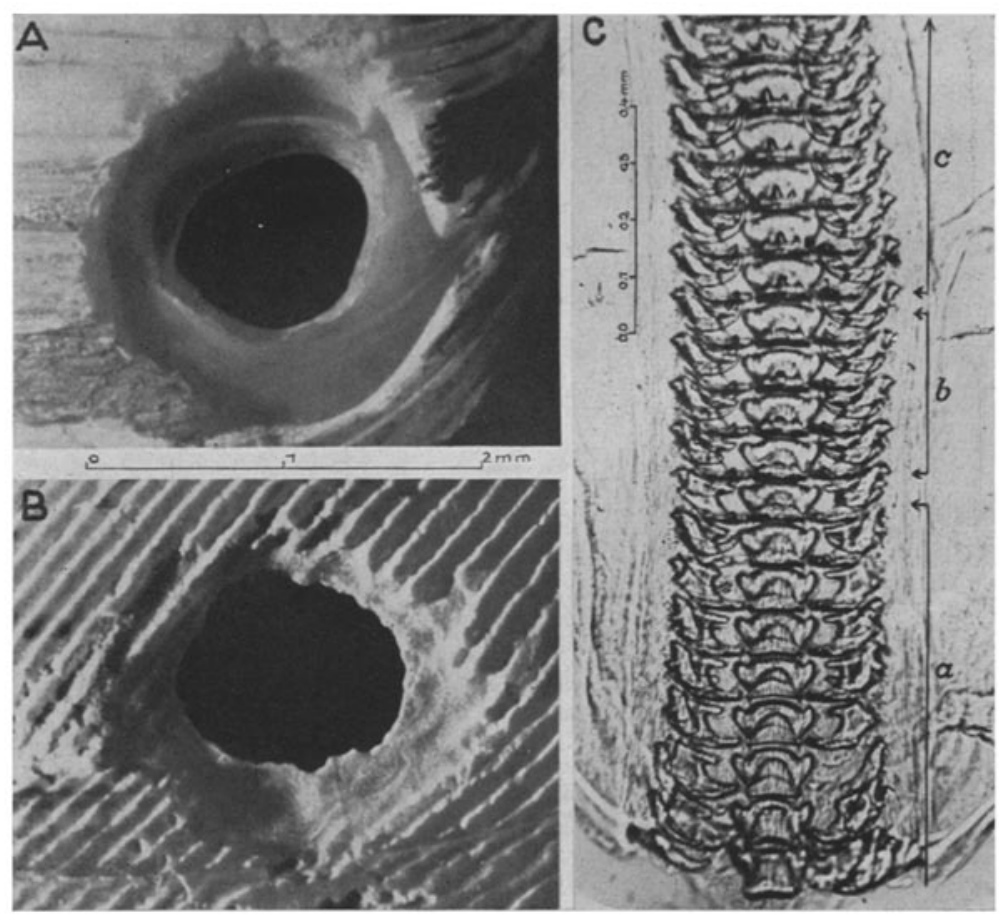

Abb. 13. $A=$ Typisches Bohrloch in einer Schale von Aloidis gibba. Nach Beendigung des Bohraktes wurde die Lunatia von der Muschel entfernt. Unmittelbar danach bohrte die Schnecke eine Angulus fabula an (B), von der sie ebenfalls, ohne gefressen zu haben, heruntergenommen wurde. $\mathrm{C}=$ Mikroaufnahme der Radula, die aus der Schnecke, nach Fertigstellung der beiden Bohrlöcher, herauspräpariert wurde. a) unterer und ältester Teil der Radula mit sehr stark abgenutzten Mittel-, Seiten- und Randzähnen. b) Knickkantenbereich, Mittelzähne weniger stark abgenutzt, c) nicht wirksamer hinterer Radulabereich mit unverbrauchten Zähnen. (Verf. phot.)

In der Abb. 13 ist das Ergebnis eines Versuches dargestellt, bei dem das Höchstmaß von Abnutgung der Radula erreicht werden konnte: Einer Lunatia (Gehäusehöhe $=10 \mathrm{~mm}$ ) wurde, nachdem sie die rechte Schale einer $9 \mathrm{~mm}$ langen Aloidis gibba durchbohrt hatte, bei Beginn des Freßaktes die Beute 
abgenommen. Unmittelbar danach nahm die Schnecke eine vorgelegte Angulus fabula (Länge $=15,5 \mathrm{~mm}$ ) sofort an und bohrte deren rechte Schale ebenfalls durch. Nach Herstellung des Bohrloches in der Angulus wurde der Lunatia die Muschel wieder abgenommen. Eine weitere Angulus griff die Schnecke nicht mehr an. Sie bewegte sich mit nicht vollkommen ausgestreckter Kriechsohle fort und lag dann mit fast geschlossenem Operculum regungslos auf dem Beckenboden. Die Präparation der Schnecke ergab schließlich die sehr stark abgenutzte Radula (Abb. 13 C), mit der das Tier nicht mehr imstande war, weitere Muscheln anzubohren. Bemerkenswert ist die Form des Bohrloches in der Angulusschale (Abb. 13 B). Die Unebenheiten in der sehr flach stehenden Wandung zeigen den sparsamen, beinahe kraftlosen und unsicheren Einsatz der Radula durch die Lunatia, die nach dem erheblichen Energieverbrauch, die das Durchbohren von Aloidisschalen mit $Z$ wischenschicht erfordert, wobei die Zähne bereits stark abgenutzt werden, keine Nahrung aufgenommen hatte.

\section{Zusammenwirken der Pharynxteile}

\section{Beim Bohren}

Die Links- oder Rechtsdrehung des Rüssels um seine Achse vor jeder Raspelperiode (s. S. 16) bewirkt die äußere Ringmuskelschicht des Hautmuskelschlauchs. Bei der dann folgenden Senkung des Pharynx ziehen die über und unter dem Schlundkopf an der Innenwand der Rüsselscheide inserierenden Muskeln den Pharynx nach vorn und drücken ihn gegen die Mundöffnung fest auf die zu bearbeitende Unterlage. Gleichzeitig dreht sich die Radulastütze in die Mundhöhle (Abb. 14 A), deren Seitenwände mit den kutikularen Platten unten auseinanderweichen. Während der Senkung und Drehung der Radulastütze drücken die Zungenknorpel mit ihren in den Hohlraum der Radulastütze ragenden, freistehenden Enden nach vorn. Dadurch wird die Knickkante um 4-5 Glieder nach dem Radulaende hin verlagert. Die Radulastütze dreht sich soweit in die Mundhöhle hinein, daß die Knickkante auf die Unterlage trifft. Das Raspeln erfolgt nun durch die Rückdrehung der Radulastütse (Abb. 14 B). Die freien Enden der Zungenknorpel bewegen sich nach der hinteren Wand zurück, dadurch verlagert sich die Knickkante ebenfalls wieder um 4-5 Radulaglieder nach hinten. Dabei spreizen sich die Randzähne nacheinander, die Mittel- und Seitenzähne der Radulaglieder an der Knickkante, und die ihr unmittelbar folgenden raspeln über den Untergrund. Die sich nacheinander spreizenden Randzähne treffen mit ihren nach oben gebogenen Spigen beim Bohrvorgang nicht auf die Raspelfläche. Nur gelegentlich zeichnen sich auf dem Grunde von unfertigen Bohrlöchern die Spuren der an der Knickkante gespreizten Randzähne ab, dünne kurze Linien laufen von der breiteren Furche der Mittelzähne in der Arbeitsrichtung der Radula schräg nach vorn (Abb. 4B). Bei den vor der Knickkante raspelnden Radulagliedern werden die Randzähne so an die Basalmembran gedrückt, daß sie mit ihren lang ausgezogenen, gebogenen Spitzen zwischen Mittelzahn und Seitenzähne des nächstfolgenden Gliedes liegen (Abb. 9 und 10G). Dadurch können die aus der Basalmembran herausragenden gezähnten Teile der Mittel- und Seitenzähne voll zur Wirkung kommen.

Dic sich im Knickkantenbereich spreizenden und wieder anlegenden Randzähne halten beim Zurückdrehen der Radulastüte abgeschabtes Schalenmaterial fest (Abb. 14 C) und befördern es so zum Ösophaguseingang. Bei der Senkung und der durch den Druck der Zungenknorpel eintretenden Verlagerung der 

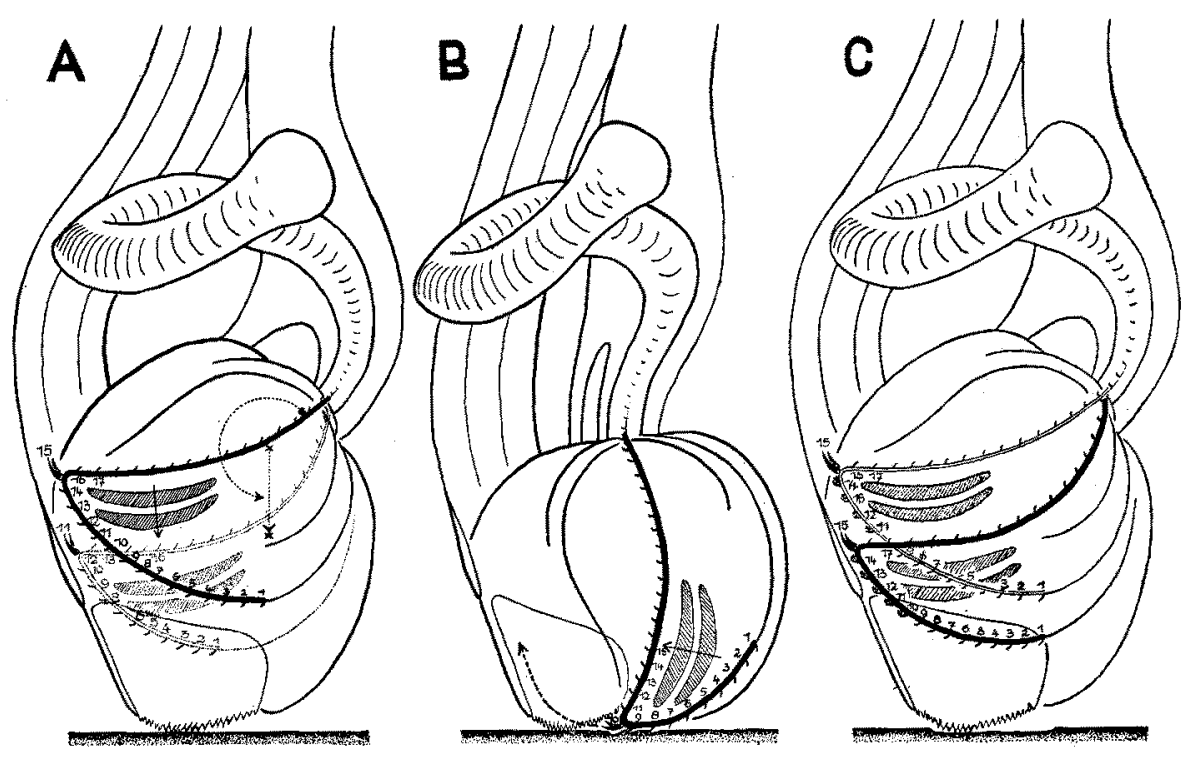

Abb. 14. Schematische Darstellung des Zusammenwirkens der Pharynxteile beim Bohrakt. Die Bezeichnungen der einzelnen Teile sind aus der Abb. 10A zu ersehen, Hautmuskelschlauch und Ganglien sind der besseren Übersicht wegen nicht mit eingezeichnet worden. Stark ausgezogen die Radulabasalmembran auf der Radulastütze, schräg schraffiert die Zungenknorpel im Querschnitt, deren Größe bei sämtlichen Phasen beibehalten wurde. A = Stellung der Radulastütze in der Ruhe (stark ausgezeichnete Radulamembran). Bei Beginn der Senkung drücken die Zungenknorpel auf die vordere Wand der Radulastütze. Dadurch verschiebt sich die Falte der festen Haut der Radulastütze und mit ihr die Knickkante. Wie aus der Numerierung der Radulaglieder, von denen nur die Mittelzähne und an der Knickkante die gespreizten Randzähne schematisch angedeutet sind, hervorgeht, verlagert sich die Knickkante um 5 Glieder nach vorn und trifft nach vollzogener Drehung und Senkung (punktierte Pfeile) in B mit dem 10. Radulaglied an der Knickkante auf die Unterlage. Die kutikularen Mundhöhlenplatten werden von der eingesenkten Radulastütze hinten auseinandergedrückt, was die verkürzte Einzeichnung der Platten andeuten soll. Unmittelbar nach Auftreffen der Radula auf der Unterlage erfolgt die Rückdrehung und Hebung der Radulastütze. Die Zungenkmorpel drücken in entgegengesetzter Richtung (Pfeil im schrägschraffierten Querschnitt) und verschieben die Knickkante bis zum 15. Radulaglied (gestrichelter Pfeil), wobei sich die Randzähne nacheinander spreizen und wieder zusammenlegen. Die Mittelzähne raspeln über den Untergrund. Das nach cinigen Raspelschlägen sich am Boden der Bohrung ansammelnde Material wird von den sich spreizenden Randzähnen erfaßt und wie in $\mathrm{C}$ bei den Radulagliedern 10-14 angedeutet, mitgeführt. Sobald die Radulastütze die Ausgangsstellung erreicht hat, erfolgt sofort wieder die Senkung. Durch den Druck der Zungenknorpel verschiebt sich wie bei A die Knickkante nach vorn und die sich spreizenden Randzähne geben das von ihnen festgehaltene Kratzmaterial nacheinander frei, das durch peristaltische Bewegungen der leistenförmigen Verstärkungen am Ösophaguseingang nach hinten in den O'sophagus weitertransportiert wird.

Knickkante nach vorn geben die Randzähne das von ihnen eingeklemmte Raspelmaterial nacheinander frei. Durch die peristaltischen Bewegungen der leistenförmigen Verstärkungen (Abb. $10 \mathrm{~B}$ ) werden die Schabereste aufgenommen und im Ósophagus weiter befördert. Die weißen Kalkteilchen sind bei bohrenden Schnecken (s. S. 16) im Ösophaguseingang stets unmittelbar vor einem Raspelschlag deutlich zu erkennen.

\section{Beim Freßakt}

Während die Randzähne beim Raspeln selbst weniger beteiligt sind, dafür das abgeschabte Material von der Bohrstelle fortschaffen, spielt der Spreiz- 
mechanismus an der Knickkante beim Freßvorgang eine wesentliche Rolle. Die Aufnahme der Weichteile läßt sich bei Schnecken, die durch das Bohrloch fressen, nicht verfolgen, da der Rüssel ins Innere der Muscheln eindringt. Nach Beobachtungen an Tieren, denen lebende Muscheln mit z. T. ausgebrochenen Schalen gereicht wurden, ist die Arbeitsweise der Pharynxteile beim Freßakt dieselbe wie beim Bohren. Nur die verschiedene Konsistenz des Untergrundes (Schale und Weichkörper der Beute) bedingen verschiedene Wirkungen.

Bei der Senkung des Pharynx drücken die gesägten Vorderränder der kutikularen Mundhöhlenauskleidung in den Weichkörper, der sich dadurch z. T. in die Mundhöhle hineinwölbt. Bei der Rückwärtsbewegung der Radulastütge dringen die an der Knickkante gespreizten Randzähne in das Muschelfleisch und reißen Stücke davon heraus, die auf dieselbe Art wie das Raspelmaterial an den Osophaguseingang transportiert werden. Die sich bei der Hebung der Radulastütze wieder enger zusammenlegenden Mundhöhlenwände klemmen den Weichkörperteil ein und halten ihn bis zur erneuten Senkung fest, wobei die gesägten Vorderkanten der die Mundöffnung überragenden chitinösen Mundhöhlenplatten das Festhalten unterstützen. Auf diese Weise werden von der Schnecke mit ihrem weit ins Muschelinnere eingedrungenen Rüssel wahrscheinlich auch Stücke des Weichkörpers losgerissen und dann in der oben beschriebenen Art verzehrt.

\section{Die "Bohrdrüse“}

Wie die in der vorangegangenen Darstellung beschriebenen Beobachtungsergebnisse zeigen, stellt $L$. nitida die Bohrlöcher mit der Radula, also auf mechanische Weise, her. Ein Durchätzen der Weichtierschalen mit Hilfe eines kalklösenden Sekrets aus dem auch bei $L$. nitida hinter der Mundöffnung liegenden scheibenförmigen Organ, der "Bohrdrüse“, im Sinne Schiemenz' schaltet demnach aus. Welche Funktion übt nun die Bohrdrüse beim Nahrungserwerb aus und welche Rolle spielt sie insbesondere beim Durchbohren der Weichtierschalen? Troschel, der das Organ bei den Naticiden entdeckte, bezeichnete es als „eine muskulöse Saugplatte" und er hielt es für wahrscheinlich, daß „mittels der Saugplatte das Tier seinen Rüssel auf der Oberfläche der anzugreifenden Muschel befestigt, um nun mit aller Kraft das runde Loch durch die Muschelschale bohren zu können". So nahm Troschel bereits damals an, daß dieses Organ am Bohrvorgang nur mittelbar beteiligt ist und daß, nach seiner Ausdrucksweise: „mit aller Kraft“, bei der Herstellung der Löcher wohl die Radula das Hauptwerkzeug darstellt. Wie schon erwähnt, werden nach Schiemenz die Löcher mit Hilfe einer von der Bohrdrüse ausgeschiedenen Säure geäzt; die Radula beginnt, so nahm Schremenz an, mit ihrer Tätigkeit erst beim Durchbruch des Loches und raspelt die letsten Reste ab. Simroth verglich die Bohrdrüse der Naticiden mit den Organen, die bei den Rhachiglossen „unter Wahrung der Abgabestelle, als secundäre Speicheldrüsen oder ,Glandes annexes' weit ins Innere getreten sind." Zur Bohrweise äußerte sich Simroth: „Der durch die tropfenweise ausgeschiedene Säure gelockerte Kalk wird durch die Radula weggeschabt, so daß ein kreisrundes abgedrechseltes Loch entsteht mit vorspringenden Rändern."

Ihrem Bau nach stellt die "Bohrdrüse" eine runde dicke Scheibe dar, die, einem Pilzhut mit kurzem Stiel ähnlich, am Hautmuskelschlauch des Rüssels sitgt. Die Farbe des Organs bei lebenden Schnecken ist hellgelb bis fast weiß. Sein Durchmesser beträgt bei $8-10 \mathrm{~mm}$ hohen L. nitida $=1-1,4 \mathrm{~mm}$. Das 
Verhältnis der größten Dicke zum Durchmesser ist etwa $1: 3$. Nach Schnittserien erhalten wir vom Aufbau des scheibenförmigen Organs folgendes Bild (Abb. 15): Unter einer dicken Kutikula, die im Mittelfeld der Bohrdrüse am stärksten ausgebildet ist und nach den Rändern allmählich dünner wird, füllt etwa die Hälfte des Organinneren eine Schicht engstehender, schlauchförmiger Drüsen aus, die keine Ausführgänge durch die Kutikula besitgen. Eine Sekretabgabe nach außen kann demnach nicht erfolgen. In den mit Hämatoxylin und Eosin gefärbten Schnittpräparaten erscheinen die Drüsenschläuche an den der

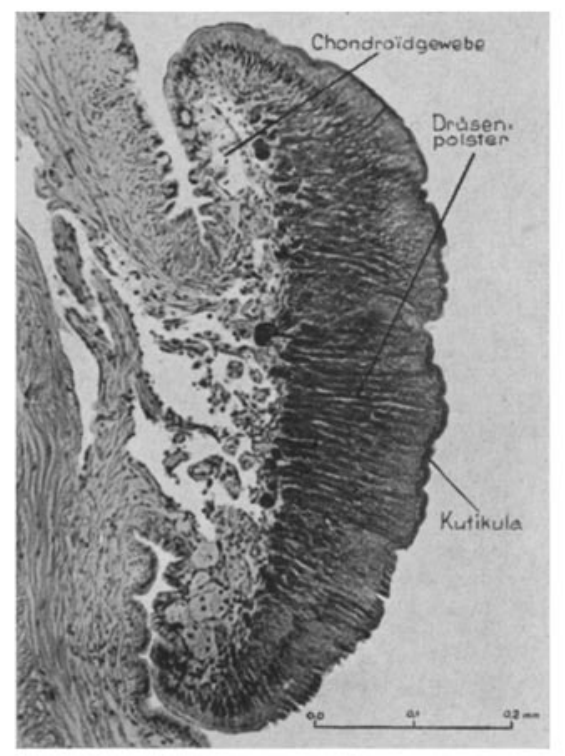

Abb. 15. Medianschnitt durch die "Bohrdrüse" von Lunatia nitida. (Färbung: HämatoxylinEosin). Bei den Rißstellen der Kutikula im unteren Teil handelt es sich nicht um Drüsenausführgänge, sondern um Beschädigungen während des Schneidens, was eindeutig daraus hervorgeht, daß die stehengebliebenen Katikulateile stets in der Verlängerung der schlauchförmigen Drüsenzellen liegen. (Verf. phot.)

Kutikula zugekehrten Enden blaß, während die Färbung nach dem Organinneren zu intensiv blauviolett wird und in den häufig zu beobachtenden am Grunde kolben- oder kugelförmig etwas aufgetriebenen Drüsenschläuchen am kräftigsten ist. Die Kerne der Drüsenzellen liegen der Kutikula genähert, etwa am Ubergang von der schwach zur stärker gefärbten Zone des Drüsenpolsters. Dem Drüsenpolster nach dem Rüssellumen zu folgt zellig-blasiges Bindegewebe, das sehr locker liegt und nach der Peripherie des Organs zu in Chondroïdgewebe übergeht, das eine Ringversteifung des Bohrdrüsenrandes bildet. An der Ringversteifung inserieren dünne Muskelfasern, die, in der Mitte des kurzen Stils sich kreuzend, an der Innenseite der Rüsselscheidenwand ansetzen und die Schnecke befähigen, den Umfang des Organs etwas zu verringern.

Obgleich die Versuche, lebenden Schnecken die Bohrdrüse operativ zu entfernen, um aus dem Verhalten der Tiere ohne Bohrdrüse Kenntnis über die Rolle, die das Organ beim Bohrakt spielt, zu erhalten, nach Anwendung aller nur möglichen Methoden bisher nicht gelangen, läßt sich nach den Beobachtungen bei $L$. nitida über die Funktion dieses Organs folgendes aussagen: In den Ruhepausen während des Bohrvorganges (s. S. 16) wird die Mundöffnung 
von der Bohrstelle abgehoben und nach vorn und oben verlagert. Dadurch kommt die Bohrdrüse so zu liegen, daß sie entweder die ganze Bohrung ausfüllt oder über ihr schwebt. Zuweilen führt in der Ruhepause der untere Rüsselteil mit der Bohrdrüse tastende Bewegungen zur Bohrstelle hin aus. Danach könnte vermutet werden, daß in der zwischen den Raspelperioden liegenden Ruhezeit ein von der Bohrdrüse ausgeschiedenes Sekret die Schalensubstanz des Beutetieres aufweicht, um der Radula das Raspeln zu ermöglichen. Das Drüsensekret müßte nach außen entleert werden, was aber wegen des Fehlens von Ausführgängen durch die Kutikula nicht möglich ist. Weiterhin beweist das Verhalten der Schnecken beim Beginn des Bohrens, daß die Radula auch ohne Mitwirkung von Lösungsmitteln die Muschel- oder Schneckenschalen bearbeiten kann, denn Lunatia fängt nach dem Ausstülpen des Rüssels sofort mit den Raspeln an (s. S. 16), und nach der ersten Raspelperiode sind deutliche Kratsspuren auf der Schalenoberfläche zu erkennen (Abb. 4 A). Nach dem gut entwickelten Drüsenpolster in der Bohrdrüse ist aber mit Sicherheit anzunehmen, daß irgendein Sekret abgesondert wird. Eine merokrine Entleerung des Drüsensekrets nach innen ist sehr wahrscheinlich. Die chemische Zusammenseţung des Sekrets konnte bisher nicht ermittelt werden, eine Säure scheidet nach den Angaben verschiedener Autoren (s. S. 2) und den Versuchen bei L. nitida aus. Die bisherigen Untersuchungen zur Ermittlung des wirksamen Stoffes in der Bohrdrüse hatten aber stets zur Grundlage, diesem Organ eine unmittelbare Beteiligung am Bohrvorgang zuzuschreiben. Die Analyse sollte ein Sekret ergeben, das den Kalk der Weichtierschalen auflöst. Das Scheitern dieser Versuche, vor allem aber die auf Grund der direkten Beobachtung bei L. ritida festgestellte mechanische Bohrweise mit Hilfe der Radula, ließen die Fragen auftauchen, ob die Absonderung der Bohrdrüse unbedingt ein die Weichtierschalen lösender Stoff sein muß und ob dieses Organ vielleicht nur indirekt am Bohrvorgang beteiligt ist. So liegt die Vermutung sehr nahe, daß ein in das Lumen der Rüsselscheide von der Bohrdrüse abgegebenes Sekret als "Schmiermittel" aufgefaßst werden kann, das die Reibung der unteren Pharynx- mit der Rüsselscheidenwand mildert. Bei der Senkung (s. S. 26) und den darauffolgenden Raspelschlägen wird der Pharynx besonders kräftig gegen den Teil der unteren Rüsselscheidenwand gezogen, an der sich die „Bohrdrüse" befindet.

Eine weitere Aufgabe, die dem nach außen unmittelbar hinter die Mundöffnung verlagerten Drüsenorgan beim Bohrvorgang wahrscheinlich zufällt, ergibt sich aus der Lebensweise der Naticiden. Die Nabelschnecken leben im Sande und überwältigen hier ihre Beute. Sie besigen keine Augen wie etwa Nucella lapillus, die Tiere der Epifauna anbohren. Bei den Naticiden ist deshalb die Tastfähigkeit gut entwickelt. Während eine Nucella, deren Fuß beim Bohrakt fest auf der Schale des Beutetieres sitzend, den arbeitenden Rüssel vermutlich allseitig umschließt, auf eine sich ihr nähernde Gefahr (durch ein größeres Tier oder anderen von der Strömung in der Gezeitenzone mitgeführten Gegenständen von der Beute heruntergerissen zu werden) einstellen kann, indem sie den Fuß noch fester anheftet (was bei bohrenden Schnecken im Aquarium bei Beschattung gut zu beobachten ist), hat eine im Sediment bohrende Lunatia, die ständig der Gefahr ausgesetgt ist, in dem von anderen Tieren der Infauna fortwährend durchwühlten Sand von ihrem Opfer abgedrängt zu werden, keine Möglichkeit, rechtzeitig durch kräftigeres Festhalten mit der Kriechsohle dagegen wirken zu können. Die Vermutung liegt daher sehr nahe, in der 
Bohrdrüse ein Organ zu sehen, mit dem Lunatia die Fähigkeit besitgt, die Bohrstelle während der zwischen den Raspelperioden liegenden Ruhepausen zu orten. Der stark auf Tastreize reagierende Rand der Bohrdrüse, was bei lebenden Schnecken, die nach dem Zertrümmern des Gehäuses den Rüssel etwas herausstrecken, durch Abtasten der Bohrdrüse mit der Spitze einer Präpariernadel gut zu beobachten ist (das Mittelfeld mit der dicken Kutikula ist vollkommen unempfindlich), läßt neben der "Ortung" der Bohrstelle als weitere Funktion dieses scheibenförmigen unteren Rüsselteils, die Fähigkeit der Orientierung über Größe und Tiefe der Bohrung vermuten, worauf die erwähnten tastenden Bewegungen des Rüsselvorderrandes während der Ruhepausen hindeuten. Damit würde auch das von verschiedenen Autoren angeführte Beweismittel, die Bohrweise mit chemischen Mitteln im Sinne von Schiemenz anzunehmen, nämlich die Übereinstimmung der Größen von Bohrloch und Bohrdrüse, die Erklärung finden.

\section{G. Zusammenfassung}

1. Bezüglich des Nahrungserwerbs sind unter den marinen Vorderkiemerschnecken die Naticiden als echte "Jäger" zu bezeichnen. Die Nabelschnecken leben im Sande und überwältigen lebende Muscheln und Schnecken, indem sie mit ihrem Rüssel die Schalen der Beutetiere durchbohren und dann den Weichkörper durch das Bohrloch ausfressen.

2. Bisher gelang es nicht, die Schnecken außerhalb des Sediments zum Bohren zu bringen, um sie bei der Bohrtätigkeit direkt zu beobachten. Deshalb konnte die Art und Weise, wie die Schnecken die Löcher herstellen, nur vermutet werden. Die am häufigsten vertretene Annahme geht auf die Untersuchungen von Schremenz zurück. Nach ihm fertigen die Nabelschnecken die Löcher mit Hilfe der "Bohrdrüse“" an, eines bei den Naticiden hinter der Mundöffnung auf der Unterseite des Rüssels liegenden Organs, das durch Absonderung einer Säure die Weichtierschalen durchätt.

3. Beobachtungen während der quantitativen Bodengreiferuntersuchungen in der Deutschen Bucht, nach denen dem Muschelmaximum im Herbst 1950 und Frühjahr 1951 ein Ansteigen der Bestandszahlen der im Untersuchungsgebiet am häufigsten vorkommenden kleinen Naticide Lunatia nitida Donovan folgte, regten zu Aquariumsversuchen an, die zunächst mit den Fragestellungen begonnen wurden, wieweit eine Bevorzugung bestimmter Muschelarten als Nahrung besteht und wie hoch das Vernichtungsmaß unter den Muscheln durch die Raubschnecken ist.

4. Das Verhalten der Schnecken bei der Fortbewegung, der Nahrungssuche, der Annahme der Beute im Aquarium, wird beschrieben.

5. Die im Aquarium von Lunatia nitida im Sand hergestellten Bohrlöcher wurden hinsichtlich ihrer Form und ihrer Lage auf den Muscheln untersucht. Kratgspuren bei unfertigen Bohrungen deuteten darauf hin, daß die Radula beim Bohrvorgang beteiligt sein muß. Eine Bevorzugung bestimmter Muschelarten als Nahrung bezüglich der Schalendicke besteht nicht, weniger angebohrt, z. T. verschmäht, wurden Muscheln mit skulptierten Schalenoberflächen wie Venus gallina oder Cardium edule.

6. Durch Anwendung verschiedener Methoden gelang es schließlich, die Schnecken über der Sandoberfläche zum Bohren zu bringen. Die direkte Beobachtung des Bohrvorganges ergab, daß Lunatia nitida die Löcher mit Hilfe 
der Radula in die Weichtierschalen bohrt. Der Bohrakt besteht aus einem Wechsel von Raspelperioden und dazwischen liegenden Ruhepausen. Die Anzahl der Schabebewegungen während der einzelnen Raspelperioden beträgt 18-20, die Ruhepausen dauern bei Beginn des Bohrens 2 bis 3 Minuten, nach $5-6$ Raspelperioden stets 5 Minuten.

7. Die Dauer des Bohraktes konnte festgestellt werden. Bei Angulus fabula benötigt L. nitida (Gehäusehöhe $=6-8 \mathrm{~mm}$ ) für die Durchbohrung von $0,1 \mathrm{~mm}$ Schalendicke rund 4 Stunden.

8. Das Aufzehren des Weichkörpers einer Muschel, deren Rohgewicht des Weichkörpers dem der fressenden Lunatia etwa entspricht, dauert $2^{1 / 2}$ Tage. Die beim Freßakt abgegebene Kotmenge besteht anfänglich aus einzelnen festen weißen Kalkspindeln, es folgen dann zusammenhängende Kotschnüre von schleimiger Konsistenz.

9. Der Bau des Lunatia-Rüssels und das Zusammenwirken der Pharynxteile beim Bohr- und Freßakt werden beschrieben und mit Hilfe von Präparationsskizzen und schematischen Zeichnungen erläutert.

10. Die „Bohrdrüse“ zeigt nach Schnittpräparaten ein gut ausgebildetes Polster von langen, engstehenden; schlauchförmigen Drüsen, die nach außen keine Ausführgänge besitzen und von einer dicken Kutikula bedeckt sind. Deshalb muß eine Abgabe des Drüsensekrets nach innen angenommen werden. Über die Funktion dieses tellerförmigen, hinter der Mundöffnung liegenden Organs läßst sich nach den Beobachtungen bei Lunatia nitida folgendes aussagen: Das ins Lumen der Rüsselscheide abgegebene Drüsensekret bildet wahrscheinlich ein "Schmiermittel", das die Reibung besonders der hinteren Pharynxwand an der inneren Rüsselscheidenwand während der kräftigen Bewegungen beim Bohr- und Freßakt mildert. Die Lage des Organs hinter der Mundöffnung und die Stellung, die es während der Ruhepausen einnimmt, ferner der auf Tastreize stark reagierende Bohrdrüsenrand lassen die Anrahme zu, daß diesem Organ als weitere Aufgabe zufällt die „Ortung" der Bohrstelle und dic Orientierung über Größe und Tiefe des Loches während des Bohraktes.

\section{H. Literatur}

Anke1, W. E. 1936 a: Prosobranchia. Grimpe-Wagler, Tierwelt d. Nord- und Ostsee IXb. - 1936 b: Die Fraßspuren von Helcion und Littorina und die Funktion der Radula. Verh. Dtsch. Zool. Ges. Freiburg i. Br. 38, 1936.

- 1937: Wie bohrt Natica? Biol. Zentralbl. 5\%.

- 1938 a: Beobachtungen an Prosobranchiern der Schwedischen Westküste. Ark. Zool. 30 A.

- 1938 b: Erwerb und Aufnahme der Nahrung bei den Gastropoden, Verh. Dtsch. Zool. Ges. 1938.

B oet tge r, C. R. 1930: Die Lage der Bohrstelle beim Angriff der Raubschnecken aus der Familie Naticidae. Z. wiss. Zool, 136.

F is ch e r, P.-H. 1922: Sur les gastropodes perceurs. Journ. d. Condhyliol. 67.

H i r s ch, G. C. 1915: Die Ernährungsbiologie fleischfressender Gastropoden. Zool. Jahrb. Abt. Allg. Zool. 35.

Jensen, Ad. S. 1951. Do the Naticidae Gastropoda (Prosobranchia) drill by chemical or by mechanical means? Vidensk. Medd. fra Dansk Naturhist. Foren. 113.

L i v a n, M. 1937: Über Bohrlöcher an rezenten und fossilen Invertebraten. Senckenbergiana 19.

Lo p pens, H. 1926: La perforation des coquilles de mollusques par les Gastropodes et des Eponges. Ann. Soc. Roy. Zool. Belgique 5\%.

Pelseneer, P. 1924: Gastropodes marins carnivores Natica et Purpura. Ann. Soc. Roy. Zool. Belgique $\mathbf{5 5}$. 
Piéron. H. 1933: Notes éthologiques sur les Gastéropodes perceurs et leur comportement. Arch. d. Zool. expérimentale et générale. Second volume jubilaire. $\mathbf{7 5}$.

Schiemenz, P. 1891: Wie bohrt Natica die Muscheln an? Mitt. Zool. Stat. Neapel 10.

S i m r oth, H. 1896-1907: Gastropoda prosobranchia. Bronn's Klassen und Ordnungen 3, 2.

Troschel, F H. 1856-1866. Das Gebiß der Schnecken. Berlin. 